Review

\title{
Biological Control of Tephritid Fruit Flies in the Americas and Hawaii: A Review of the Use of Parasitoids and Predators
}

\author{
Flávio R. M. Garcia ${ }^{1, *}$, Sérgio M. Ovruski ${ }^{2}$, Lorena Suárez ${ }^{3}$, Jorge Cancino ${ }^{4}$ and Oscar E. Liburd 5 \\ 1 Departamento de Ecologia, Instituto de Biologia, Zoologia e Genética, Universidade Federal de Pelotas, \\ Pelotas 96010900, RS, Brazil \\ 2 LIEMEN, División Control Biológico de Plagas, PROIMI Biotecnología, CCT NOA Sur-CONICET, Avda, \\ Belgrano y Pje, Caseros, San Miguel de Tucumán T4001MVB, Tucumán, Argentina; \\ sovruski@conicet.gov.ar \\ 3 Dirección de Sanidad Vegetal, Animal y Alimentos de San Juan, Av. Nazario Benavides 8000 Oeste, \\ Rivadavia CP 5400, San Juan, Argentina; ldcsuarez@sanjuan.gov.ar \\ 4 Programa Moscafrut SAGARPA-IICA, Camino a los Cacahoatales s/n, Metapa de Dominguez 30860, \\ Chiapas, Mexico; jorge.cancino.i@senasica.gob.mx \\ 5 Entomology and Nematology Department, University of Florida, 1881 Natural Area Dr., Gainesville, FL \\ 32611-0620, USA; oeliburd@ufl.edu \\ * Correspondence: flavio.garcia@ufpel.edu.br
}

Received: 31 August 2020; Accepted: 22 September 2020; Published: 25 September 2020

Simple Summary: Biological control has been the most commonly researched control tactic within fruit fly management programs, and parasitoids have been the main natural enemies used against pestiferous fruit fly species. In view of this fact, it is important to highlight and compile the data on parasitoids with a certain frequency, aiming to facilitate the knowledge of all the researchers. Information regarding the activities of parasitoids and predators on pestiferous fruit flies in the Americas is limited; therefore, this study aimed to compile the diversity of parasitoids and predators associated with tephritid fruit flies, as well as providing the scientific evidence about the use of parasitoids and predators as biological control agents for fruit flies im the Americas and Hawaii.

\begin{abstract}
Biological control has been the most commonly researched control tactic within fruit fly management programs. For the first time, a review is carried out covering parasitoids and predators of fruit flies (Tephritidae) from the Americas and Hawaii, presenting the main biological control programs in this region. In this work, 31 species of fruit flies of economic importance are considered in the genera Anastrepha (11), Rhagoletis (14), Bactrocera (4), Ceratitis (1), and Zeugodacus (1). In this study, a total of 79 parasitoid species of fruit flies of economic importance are listed and, from these, 50 are native and 29 are introduced. A total of 56 species of fruit fly predators occur in the Americas and Hawaii.
\end{abstract}

Keywords: classic biological control; conservation biological control; augmentative biological control; biological control programs

\section{Introduction}

In the Americas, there are four genera of tephritid fruit flies, which include species of economic and quarantine importance. Anastrepha Schiner, 1868 is widely distributed in the neotropical region [1], and seven species are economically important in the tropics and subtropics due to their wide range of commercial host plants and distribution. The species include Anastrepha ludens (Loew) (Mexican fruit fly), A. obliqua (Macquart) (the West Indian fruit fly), A. fraterculus (Wiedemann) (South 
American fruit fly), A. suspensa (Loew) (Caribbean fruit fly), A. serpentina (Wiedemann) (Sapotaceas fly), A. striata Schiner (guava fly), and A. grandis (Macquart) (melon fly). Rhagoletis Loew is mainly distributed in the neartic region and involves several pestiferous species such as Rhagoletis pomonella Walsh, one of the main pests of hawthorn in Central Mexico and apples in the Eastern United States, and R. mendax Curran, one of the most important blueberries pests in the Eastern United States [2]. $R$. cingulata (Loew) and its related R. fausta Osten Sacken are key pests of cherries, Prunus spp., in Eastern and Midwestern United States [3]. The native host of $R$. cingulata is the wild black cherry, $P$. serotina Ehrh, whereas $R$. fausta infests pin cherry, P. pennsylvanica L. [4]. A distant relative, $R$. completa (Cresson), infests walnuts and cause economic damage in the Western United States. There are also several neotropical Rhagoletis species associated with the Solanaceae, among which are two pestiferous species, $R$. tomatis Foote and $R$. nova (Schiner), which are associated with the tomato and the sweet cucumber, respectively [2].

Bactrocera Macquart and Ceratitis Macleay are two tephritid genera that were introduced into the continent. Two Bactrocera species are found in the Americas: B. oleae (Rossi) is present in the United States in the state of California [5], while B. carambolae Drew and Hancock is located in South America, distributed in Surinam, French Guiana, and Northern Brazil [6]. Ceratitis capitata (Wiedemann) is the only Ceratitis species in America, and it is widely distributed in Central and South America [7].

In Hawaii, C. capitata and three invasive Bactrocera species, i.e., Zeugodacus cucurbitae (Coquillett) (melon fly), Bactocera dorsalis (Hendel) (oriental fruit fly), and Bactrocera latifrons (Hendel) (Solonaceous fruit fly), are economically important and they rank high on quarantine lists worldwide [8].

Several natural enemies have been associated with tephritid fruit flies in the Americas and Hawaii either naturally occurring or through laboratory, field cages, and open-field testing with introduced or native species [9-14]. Biological control has been the most commonly researched control tactic within fruit fly management programs, and parasitoids have been the main natural enemies used against pestiferous fruit fly species [8,13]. In view of this fact, it is important to highlight and compile the data on parasitoids with a certain frequency, aiming to facilitate the knowledge to facilitate research in this field. The most recent and comprehensive review on the biological control of fruit flies using parasitoids was conducted 20 years ago covering Latin America and southern regions of the United States [11]. Since then, the main articles that reviewed augmentative biological control using parasitoids were from Mexico [15], Argentina [16], and Brazil [17]. Both Americannative and exotic parasitoids are currently considered as candidates for biological control programs in the Americas [13,18-20]. The most recent reviews of parasitoids used in biological control programs in Hawaii were published by Bokonon-Ganta et al. [21] and Vargas et al. [8].

The use of entomopathogenic fungi and nematodes against fruit flies has shown promising results, which were recently reviewed by Dias et al. [13]. However, predators have been underexplored as control agents of fruit flies [11,22,23], although they are potentially important in conservation biological control [24,25]. Fruit fly immature stages are naturally exposed to a variety of predators, among which the most important are ants (Formicidae) and rove beetles (Staphylinidae), which have been detected to cause significant mortality $[22,23,25,26]$. Therefore, information on predator interactions with other biological control agents is the basis of studies on intraguild predation in augmentative biological control [27] and the compatibility between augmentative and conservation biological control in an approach for integrated pest management.

In this work, 29 species of fruit flies of economic importance are considered. They belong to the genera Anastrepha (12), Rhagoletis (10), Bactrocera (5), Ceratitis (1), and Zeugodacus (1), and are the following: A. curvicauda (Gerstaecker), A. distincta Greene, A. fraterculus (Wied.), A. grandis (Macquart), A. ludens Loew, A. obliqua (Macquart), A. pseudoparallela Loew, A. pickeli Lima, A. serpentina (Wied), A. sororcula Zucchi, A. striata Schiner, A. suspensa (Loew), B. carambolae Drew and Hancock, B. dorsalis (Hendel), B. latifrons (Hendel), B. oleae (Rossi), C. capitata (Wied.), R. cerasi (L.), R. completa Cresson, $R$. cingulata (Loew), R. fausta (Osten Sacken), R. ferruginea (Hendel), R. mendax (Curran), R. meiiginii (Loew), R. pomonella (Walsh), R. suavis (Loew), R. tabellaria (Fitch), R. zephyria Snow, and Z. curcubitae (Coquillett). 
Information regarding the activities of parasitoids and predators on pestiferous fruit flies in the Americas is limited; therefore, this study aimed to compile a list of the diversity of parasitoid and predator species associated with tephritid fruit flies, as well as providing scientific evidence about the use of parasitoids and predators as biological control agents for fruit flies

\section{Parasitoids}

The parasitoids that deposit an egg in the host are either solitary, more than one, or gregarious. When the parasitoids develop inside the host, they are endoparasitoids, and when development occurs externally, they are ectoparasitoids. Parasitoids can be either idiobionts or koinobionts. The former refers to those who kill their hosts shortly after oviposition, preventing further development, while the latter characterizes those who allow develop in the living hosts and kill them at the end of their cycle [28].

\subsection{Native Parasitoids}

In the Americas, there is a richness of 51 species of native parasitoids of fruit flies (Diptera, Tephritidae) of frugivorous fruit flies with economic importance included in 20 genera of 7 families (Braconidae, Diapriidae, Eurytomidae, Figitidae, Ichneumonidae, Mymaridae, and Pteromalidae). The Braconidae and Figitidae account for $64.7 \%$ of the fruit fly parasitoid species on the American continent. The family with the highest species richness is the Braconidae, with $47.1 \%$ of the total, followed by Figitidae with $17.6 \%$, Pteromalidae with $17.6 \%$, and Diapriidae with $11.8 \%$. Ichneumonidae, Eurytomidae, and Mymaridae are represented by only one species each $(5.9 \%$ one species each).

Most parasitoid species (53\%) are associated with the genus Anastrepha, while 35.3\%, 31.4\%, and $7.8 \%$ of all parasitoid species are associated with Rhagoletis, Ceratitis, and Bactrocera, respectively. Table 1 shows the relationship between the parasitoid genera and the tephritid genera.

Table 1. Association between the genus of native parasitoids (Hymenoptera) and the genus of fruit flies with economic importance in the Americas.

\begin{tabular}{|c|c|c|c|c|}
\hline \multirow[t]{2}{*}{ Parasitoid Species } & \multicolumn{4}{|c|}{ Genus of Tephritidae } \\
\hline & Anastrepha & Bactrocera & Ceratitis & Rhagoletis \\
\hline \multicolumn{5}{|l|}{ Braconidae } \\
\hline Asobara & $x$ & & $x$ & \\
\hline Diachasma & & & & $x$ \\
\hline Diachasmimorpha & & & & $x$ \\
\hline Doryctobracon & $x$ & & $x$ & \\
\hline Eurytenes & & & & $x$ \\
\hline Opius & $x$ & $x$ & $x$ & $x$ \\
\hline Utetes & $x$ & & & $x$ \\
\hline \multicolumn{5}{|l|}{ Diapriidae } \\
\hline Coptera & $x$ & $x$ & $x$ & $x$ \\
\hline Trichopria & $X$ & & & \\
\hline \multicolumn{5}{|l|}{ Eurytomidae } \\
\hline Eurytoma & $x$ & & & \\
\hline \multicolumn{5}{|l|}{ Figitidae } \\
\hline Aganaspis & $x$ & & $x$ & $x$ \\
\hline Dicerataspis & $x$ & & & \\
\hline Lopheucoila & $X$ & & & \\
\hline Odontosema & $\mathrm{X}$ & & $x$ & \\
\hline Tropideucoila & $x$ & & & \\
\hline Rhoptromeris & $x$ & & & \\
\hline Ichneumonidae & & & & $x$ \\
\hline
\end{tabular}




\begin{tabular}{ccc}
\hline Phygadeuon & & \\
Mymaridae & & \\
Anaphes & & \\
Pteromalidae & $\mathrm{x}$ & $\mathrm{x}$ \\
Pteromalus & $\mathrm{x}$ & $\mathrm{x}$ \\
Spalangia & \\
\hline
\end{tabular}

Dicerataspis Ashmead, Eurytoma Illiger Lopheucoila Weld, Trichopria Ashmead, Tropideucoila Ashmead, and Rhoptromeris Förster are associated exclusively with Anastrepha, while Diachasma Foerster, Diachasmimorpha Viereck, and Eurytenes Foerster are solely associated with Rhagoletis. The exotic genera of fruit flies, Bactrocera and Ceratitis, have no exclusive genus of parasitoid. Doryctobracon Enderlein, with a greater species richness, is associated with both Anastrepha and Ceratitis. Coptera Say and Opius Wesmael are the only genus of parasitoids associated with the 4 genera of tephritid fruit flies of economic importance.

Twenty host species associated with the Braconidae (Anastrepha (11 spp.); Rhagolethis (8 spp.); Ceratitis (1 sp.), were recorded. The Figitidae has 11 associated host species belonging to Anastrepha (7 spp.), Rhagolethis (3 spp.), and Ceratitis (1 sp.). The Diapriidae has 12 hosts species belonging to Rhagoletis (6 spp.), Anastrepha (5 spp.), and Ceratitis (1 sp.). The Pteromalidae has 6 hosts species Anastrepha (3 spp.), Bactrocera (2 spp.), Ceratitis (1 sp.). The Eurytomidae has 3 hosts (all Anastrepha). The Ichneumonidae has 2 hosts (all Rhagoletis), and Mymaridae with 1 (Rhagoletis) (Table 2).

Table 2. Native parasitoid species and their fruit fly hosts in the Americas.

\begin{tabular}{|c|c|c|}
\hline Parasitoid Species & Host Fruit Fly Species & References \\
\hline Braconidae & & \\
\hline Asobara anastrephae & A. fraterculus, A. obliqua, A. striata, C. capitata & {$[17,29,30-36]$} \\
\hline Diachasma alloeum & R. pomonella, R. mendax, R. zephyria & [37] \\
\hline Diachasma ferrugineum & R. pomonella & [38] \\
\hline $\begin{array}{l}\text { Diachasmimorpha } \\
\text { martinalujai }\end{array}$ & R. pomonella & [39] \\
\hline $\begin{array}{l}\text { Diachasmimorpha } \\
\text { mellea }\end{array}$ & R. cingulata, $R$. mendax, R. pomonella, $R$. zephyria & {$[37,39,40]$} \\
\hline $\begin{array}{l}\text { Diachasmimorpha } \\
\text { sublaevis }\end{array}$ & R. completa & [41] \\
\hline Doryctobracon adaimei & A. fraterculus, A. striata & {$[35,42]$} \\
\hline Doryctobracon areolatus & $\begin{array}{c}\text { A. distincta, A. fraterculus, A. ludens, A. obliqua, A. pseudoparallela, } \\
\text { A. pickeli, A. serpentina, A. sororcula, A. striata, A. suspensa, C. } \\
\text { capitata }\end{array}$ & {$[17,29,30,43-62]$} \\
\hline $\begin{array}{l}\text { Doryctobracon } \\
\text { auripennis }\end{array}$ & A. serpentina & [55] \\
\hline $\begin{array}{l}\text { Doryctobracon } \\
\text { brasiliensis }\end{array}$ & A. fraterculus, A. obliqua, A. pickeli, A. serpentina, A. sororcula & {$[17,43,44,48,59,61,63-65]$} \\
\hline Doryctobracon capsicola & A. serpentina & [55] \\
\hline $\begin{array}{l}\text { Doryctobracon } \\
\text { crawfordi }\end{array}$ & $\begin{array}{c}\text { A. distincta, A. fraterculus, A. ludens, A. obliqua, A. serpentina, A. } \\
\text { sororcula, A. striata, C. capitata }\end{array}$ & $\begin{array}{c}{[17,29,47,49,50,52,53,55,58,59,64,} \\
66-68]\end{array}$ \\
\hline $\begin{array}{l}\text { Doryctobracon } \\
\text { fluminensis }\end{array}$ & A. fraterculus, A. pickeli, A. pseudoparallela & {$[17,43,44,50]$} \\
\hline $\begin{array}{l}\text { Doryctobracon } \\
\text { toxotrypanae }\end{array}$ & A. curcuvicauda & [69] \\
\hline $\begin{array}{l}\text { Doryctobracon } \\
\text { trinidadensis }\end{array}$ & A. serpentina, A. striata & {$[29,70]$} \\
\hline Doryctobracon zeteki & A. serpentina, A. striata & {$[29,45,50,55]$} \\
\hline $\begin{array}{c}\text { Eurytenes } \\
\text { (Stigmatopoea) maya }\end{array}$ & R. pomonella & [39] \\
\hline Opius bellus & $\begin{array}{c}\text { A. distincta, A. fraterculus, A. obliqua, A. pickeli, A. serpentina, A. } \\
\text { sororocula, A. striata, C. capitata, } R \text {. ferrugínea }\end{array}$ & $\begin{array}{c}{[17,29-} \\
32,43,44,48,54,59,60,63,65,71-75]\end{array}$ \\
\hline Opius downesi & R. pomonella, R. tabellaria, R. zephyria & [37] \\
\hline Opius hirtus & A. curvicauda, A. ludens, A. obliqua & {$[18,76-79]$} \\
\hline Utetes anastrephae & $\begin{array}{l}\text { A. distincta, A. fraterculus, A. ludens, A. obliqua, A. pickeli, A. } \\
\text { serpentina, A. sorocula, A. striata, A. suspensa, C. capitata }\end{array}$ & $\begin{array}{c}{[17,18,30,43,44,46-} \\
48,50,52,56,59,60-63,66,75,80-87]\end{array}$ \\
\hline Utetes canaliculatus & R. pomonella & {$[37,69,88,89]$} \\
\hline
\end{tabular}




\begin{tabular}{|c|c|c|}
\hline Utetes lectoides & R. pomonella & [37] \\
\hline Utetes richmondi & R. carnivora, R. mendax & {$[37,69]$} \\
\hline $\begin{array}{l}\text { Utetes tomoplagiae (as } \\
\text { Opius tomoplagiae) }\end{array}$ & A. fraterculus & {$[17,90-92]$} \\
\hline \multicolumn{3}{|l|}{ Diapriidae } \\
\hline Coptera cingulatae & R. cingulata, $R$. fausta, R. pomonella, $R$. suavis & [69] \\
\hline Coptera evansi & R. completa, $R$. fausta & {$[41,69]$} \\
\hline Coptera haywardi & $\begin{array}{c}\text { A. fraterculus, A. ludens, A. obliqua, A. serpentina, A. sororcula, } C . \\
\text { capitata }\end{array}$ & {$[16-18,52,93-98]$} \\
\hline Coptera pomonellae & R. pomonella, $R$. suavis & [69] \\
\hline Coptera occidentalis & R. cingulata, R. cerasi, R. completa & {$[41,69]$} \\
\hline $\begin{array}{l}\text { Trichopria anastrephae } \\
\text { Eurytomidae }\end{array}$ & A. fraterculus, A. obliqua, A. serpentina, C. capitata & {$[17,48,98-100]$} \\
\hline $\begin{array}{l}\text { Eurytoma sivinskii } \\
\quad \text { Figitidae }\end{array}$ & A. ludens, A. obliqua, A. serpentina & [101] \\
\hline Aganaspis alujai & R. completa, R. ramosae, R. zoqui & {$[102,103]$} \\
\hline Aganaspis nordlanderi & A. fraterculus, A. striata, C. capitata & {$[17,104,105]$} \\
\hline Aganaspis pelleranoi & $\begin{array}{l}\text { A. distincta, A. fraterculus, A. ludens, A. obliqua, A serpentina, A. } \\
\text { striata, C. capitata }\end{array}$ & $\begin{array}{c}{[17,18,44,50,52,56,59,61,66,104-} \\
106]\end{array}$ \\
\hline $\begin{array}{l}\text { Dicerataspis } \\
\text { grenadensis }\end{array}$ & A. fraterculus & {$[17,107]$} \\
\hline Lopheucoila anastrephae & A. fraterculus, A. pseudoparallela & {$[17,34,59,105]$} \\
\hline $\begin{array}{l}\text { Odontosema } \\
\text { anastrephae }\end{array}$ & A. fraterculus, A. ludens, A. obliqua, C. capitata & $\begin{array}{c}{[17,34,52,56,59,66,75,104,105,108,} \\
109]\end{array}$ \\
\hline $\begin{array}{l}\text { Odontosema albinerve } \\
\text { Tropideucoila weldi }\end{array}$ & $\begin{array}{l}\text { A. fraterculus. A. serpentina, C. capitata } \\
\text { A. fraterculus, A. sororcula }\end{array}$ & $\begin{array}{c}{[48,110]} \\
{[111]}\end{array}$ \\
\hline $\begin{array}{l}\text { Rhoptromeris haywardi } \\
\text { Ichneumonidae }\end{array}$ & A. fraterculus, C. capitata & {$[16]$} \\
\hline $\begin{array}{l}\text { Phygadeuon wiesmanni } \\
\text { Mymaridae }\end{array}$ & R. pomonella, R. cerasi & [112] \\
\hline $\begin{array}{l}\text { Anaphes conotracheli } \\
\text { Pteromalidae }\end{array}$ & R. pomonella & [112] \\
\hline Pteromalus kapaunae & B. oleae & {$[69,113]$} \\
\hline Spalangia cameroni & Z. curcubitae & [69] \\
\hline Spalangia endius & A. fraterculus, A. obliqua. C. capitata & [98] \\
\hline Spalangia gemina & A. fraterculus, A. obliqua, C. capitata & {$[93,98,114]$} \\
\hline Spalangia impunctata & C. capitata & [98] \\
\hline Spalangia leiopleura & C. capitata & [98] \\
\hline Spalangia nigra & Z. cucurbitae & [69] \\
\hline Spalangia simplex & A. serpentina, C. capitata & [98] \\
\hline
\end{tabular}

The native parasitoid species that has the largest number of hosts is Doryctobacon areolatus with 11 hosts (Anastrepha spp. and Ceratitis capitata), followed by Utetes anastrephae with 10 hosts (Anastrepha spp. and Cerattis capitata) (Table 2). Anastrepha grandis is known as the South American cucurbit fruit fly, a quarantine pest and the main fruit fly pest of cucurbitaceous plants that has no parasitoid associated. The absence of knowledge of natural enemies of A. grandis (parasitoids and predators) is probably due to the following factors: (1) Few studies carried out with this species when compared with other species of fruit flies of economic importance, (2) parasitoids have difficulty ovipositing in A. grandis larvae and eggs because the ovipositors of native parasitoid species may be unable to pierce the thick epicarp of the host fruits of this fly (Cucurbitaceae), (3) the absence of studies of pupae parasitodes and predators of $A$. grandis.

Parasitoids of economic fruit flies have been recorded in 20 of the 35 countries that are part of the Americas. Brazil has 19 native parasitoids and continental USA has 15 parasitoids, followed by Argentina and Mexico with 14 each (Table 3). The greatest occurrence of parasitoids in these countries is mainly due to the fact of a higher intensity of fruit fly parasitoid surveys compared to other countries. 
Table 3. List and distribution of native Hymenopteran parasitoid on fruit-infesting Tephritidae in the Americas.

\begin{tabular}{|c|c|}
\hline Native Parasitoid Species & Countries \\
\hline \multicolumn{2}{|l|}{ Braconidae } \\
\hline Asobara anastrephae & ARG, BRA, COL, PAN \\
\hline Diachasma alloeum & USA \\
\hline D. ferrugineum & USA \\
\hline $\begin{array}{l}\text { Diachasmimorpha } \\
\text { martinalujai }\end{array}$ & MEX \\
\hline D. sublaevis & USA \\
\hline D. mellea & MEX, USA \\
\hline Doryctobracon adaimei & BRA \\
\hline D. areolatus & ARG, BOL, BRA, COL, COR, ECU, ELS, FRG, GUA, MEX, PAN, USA, VEN \\
\hline D. auripennis & PAN \\
\hline D. brasiliensis & ARG, BOL, BRA \\
\hline D. capsicola & PAN \\
\hline D. crawfordi & ARG, BOL, BRA, COL, COR, ECU, ELS, GUA, MEX, PAN, VEN \\
\hline D. fluminensis & BRA, VEN \\
\hline \multirow[t]{2}{*}{ D. toxotrypanae } & COR, ELS \\
\hline & MEX, VEN \\
\hline D. trinidadensis & PAN, TRT \\
\hline D. zeteki & COL, PAN, VEN \\
\hline $\begin{array}{c}\text { Eurytenes (Stigmatopoea) } \\
\text { maya }\end{array}$ & MEX \\
\hline Opius bellus & ARG. BEL, BOL. BRA, COR, FRG, MEX, PAN, VEN \\
\hline O. downesi & CAN, USA \\
\hline O. hirtus & COR, DOR, MEX, \\
\hline Utetes anastrephae & $\begin{array}{c}\text { ARG, BOL, BRA, COL, COR, CUB, ECU, ELS, FRG, GUA, MEX, PUR, USA, } \\
\text { VEN }\end{array}$ \\
\hline U. canaliculatus & CAN, USA, MEX \\
\hline U. richmondi & CAN, USA \\
\hline U. lectoides & CAN, USA \\
\hline \multicolumn{2}{|l|}{ Diapriidae } \\
\hline Coptera cingulatae & USA \\
\hline C. evansi & USA \\
\hline C. haywardi & ARG. BRA. MEX, VEN \\
\hline C. pomonellae & USA \\
\hline C. occidentalis & USA \\
\hline Trichopria anastrephae & ARG, BRA, VEN \\
\hline \multicolumn{2}{|l|}{ Eurytomidae } \\
\hline Eurytoma sivinskii & MEX \\
\hline \multicolumn{2}{|l|}{ Figitidae } \\
\hline Aganaspis alujai & MEX, USA \\
\hline A. nordlanderi & ARG, BRA, COR \\
\hline A. pelleranoi & ARG, BOL, BRA, COL, COR, ECU, FRG, GUA, MEX, PER, VEM \\
\hline Dicerataspis grenadensis & ARG \\
\hline Lopheucoila anastrephae & ARG, BOL, BRA, PAN \\
\hline Odontosema anastrephae & ARG, BOL, BRA, COL, COR, MEX \\
\hline O. albinerve & BRA \\
\hline Tropideucoila weldi & BRA \\
\hline \multicolumn{2}{|l|}{ Ichneumonidae } \\
\hline \multicolumn{2}{|l|}{ Mymaridae } \\
\hline $\begin{array}{l}\text { Anaphes conotracheli } \\
\text { Pteromalidae }\end{array}$ & CAN \\
\hline Pteromalus kapaunae & USA \\
\hline
\end{tabular}




\begin{tabular}{cc}
\hline Spalangia cameroni & USA \\
S. endius & USA, BRA \\
S. gemina & BRA \\
S. impunctata & BRA \\
S. leiopleura & BRA \\
S. nigra & USA \\
S. simplex & BRA \\
\hline
\end{tabular}

Countries: ARG, Argentina; BEL, Belize; BOL, Bolivia; BRA, Brazil; CAN, Canada; COL, Colombia; COR, Costa Rica; CUB, Cuba; DOR, Dominican Republica; ECU, Ecuador; ELS, El Salvador; FRG, French Guiana; GUA, Guatemala; MEX, Mexico; PAN, Panama; PER, Peru; PUR, Puerto Rico, TRT, Trinidad and Tobago; VEN, Venezuela; USA, United States of America.

Most native parasitoids are solitary $(81.1 \%)$, koinobionts $(62.3 \%)$, or endoparasitoids $(68.8 \%)$. The native species of Braconidae and Figitidae include endoparasitoid koinobionts that attack mainly larvae. The species of parasitoid idiobionts belong to the Diapriidae, Eurytomidae, and Pteromalidae. Ectoparasitism is found in the Eurytomidae and Pteromalidae. Most native parasitoids attack the larval stage $(56.4 \%)$, pupa (32. $0 \%)$, and eggs $(12.0 \%)$. There is no native parasitoid with a gregarious habit (Table 4).

Table 4. Guilds of native parasitoids of tephritid fruit flies with economic importance in the Americas.

\begin{tabular}{|c|c|c|c|}
\hline Native Parasitoid Species & Parasitism Modes & Feeding Types & Host Stage Attacked \\
\hline \multicolumn{4}{|l|}{ Braconidae } \\
\hline Asobara anastrephae & $\mathrm{S}, \mathrm{K}$ & En & L3 \\
\hline Diachasma alloeum & U & En & $\mathrm{L}$ \\
\hline D. ferrugineum & $\mathrm{U}$ & $\mathrm{U}$ & $\mathrm{L}$ \\
\hline Diachasmimorpha martinalujai & S, K & En & $\mathrm{L}$ \\
\hline D. mellea & S, K & En & $\mathrm{L}$ \\
\hline D. sublaevis & $\mathrm{S}, \mathrm{K}$ & En & $\mathrm{L}$ \\
\hline Doryctobracon adaimei & S, K & En & $\mathrm{L}$ \\
\hline D. areolatus & S, K & En & E-L1 \\
\hline D. auripennis & S, K & En & $\mathrm{L}$ \\
\hline D. brasiliensis & S, K & En & L3 \\
\hline D. capsicola & $\mathrm{S}, \mathrm{K}$ & En & $\mathrm{L}$ \\
\hline D. crawfordi & S, K & En & L3 \\
\hline D. fluminensis & S, K & En & $\mathrm{L}$ \\
\hline D. toxotrypanae & S, K & En & $\mathrm{L}$ \\
\hline D. trinidadensis & $S, K$ & En & $\mathrm{L}$ \\
\hline D. zeteki & $\mathrm{S}, \mathrm{K}$ & En & $\mathrm{L}$ \\
\hline Eurytenes (Stigmatopoea) maya & $\mathrm{U}$ & $\mathrm{U}$ & $\mathrm{L}$ \\
\hline Opius bellus & S, K & En & L2-L3 \\
\hline O. downesi & S, K & En & $\mathrm{L}$ \\
\hline O. hirtus & S, K & En & $\mathrm{L}$ \\
\hline Utetes anastrephae & S, K & En & L3 \\
\hline U. canaliculatus & $\mathrm{U}$ & En & $\mathrm{E}$ \\
\hline U. richmondi & $\mathrm{U}$ & En & E \\
\hline U. lectoides & $\mathrm{U}$ & $\mathrm{U}$ & E \\
\hline \multicolumn{4}{|l|}{ Diapriidae } \\
\hline Coptera cingulatae & $S, I$ & En & $\mathrm{P}$ \\
\hline C. evansi & $S, I$ & En & $\mathrm{P}$ \\
\hline C. haywardi & $S, I$ & En & $\mathrm{P}$ \\
\hline C. pomonellae & $S, I$ & En & $\mathrm{P}$ \\
\hline C. occidentalis & $S, I$ & En & $\mathrm{P}$ \\
\hline Trichopria anastrephae & S, I & En & $\mathrm{P}$ \\
\hline
\end{tabular}




\begin{tabular}{cccc}
\hline $\begin{array}{c}\text { Eurytomidae } \\
\text { Eurytoma sivinskii } \\
\text { Figitidae }\end{array}$ & $\mathrm{S}, \mathrm{I}$ & $\mathrm{Ec}$ & $\mathrm{P}$ \\
Aganaspis alujai & $\mathrm{S}, \mathrm{K}$ & $\mathrm{En}$ & $\mathrm{L} 3$ \\
A. pelleranoi & $\mathrm{S}, \mathrm{K}$ & $\mathrm{En}$ & $\mathrm{L} 3$ \\
A. nordlanderi & $\mathrm{S}, \mathrm{K}$ & $\mathrm{En}$ & $\mathrm{L} 3$ \\
Dicerataspis flavipes & $\mathrm{S}, \mathrm{K}$ & $\mathrm{En}$ & $\mathrm{L} 3$ \\
D. grenadensis & $\mathrm{S}, \mathrm{K}$ & $\mathrm{En}$ & $\mathrm{L} 3$ \\
Lopheucoila anastrephae & $\mathrm{S}, \mathrm{K}$ & $\mathrm{En}$ & $\mathrm{L} 3$ \\
Odontosema anastrephae & $\mathrm{S}, \mathrm{K}$ & $\mathrm{En}$ & $\mathrm{L} 3$ \\
O. albinerve & $\mathrm{S}, \mathrm{K}$ & $\mathrm{En}$ & $\mathrm{L} 3$ \\
Tropideucoila weldi & $\mathrm{S}, \mathrm{K}$ & $\mathrm{En}$ & $\mathrm{L}$ \\
Rhoptromeris haywardi & $\mathrm{S}, \mathrm{K}$ & $\mathrm{En}$ & $\mathrm{L}$ \\
Ichneumonidae & & & \\
Phygadeuon wiesmanni & $\mathrm{U}$ & $\mathrm{U}$ & $\mathrm{P}$ \\
Mymaridae & & & $\mathrm{E}$ \\
Anaphes conotracheli & $\mathrm{U}$ & $\mathrm{U}$ & \\
Pteromalidae & & & $\mathrm{P}$ \\
Pteromalus kapaunae & $\mathrm{S}, \mathrm{I}$ & $\mathrm{Ec}$ & $\mathrm{P}$ \\
Spalangia cameroni & $\mathrm{S}, \mathrm{I}$ & $\mathrm{Ec}$ & $\mathrm{P}$ \\
S. endius & $\mathrm{S}, \mathrm{I}$ & $\mathrm{Ec}$ & $\mathrm{P}$ \\
S. gemina & $\mathrm{S}, \mathrm{I}$ & $\mathrm{Ec}$ & $\mathrm{P}$ \\
S. impunctate & $\mathrm{S}, \mathrm{I}$ & $\mathrm{Ec}$ & $\mathrm{P}$ \\
S. leiopleura & $\mathrm{S}, \mathrm{I}$ & $\mathrm{Ec}$ & $\mathrm{P}$ \\
S. nigra & $\mathrm{S}, \mathrm{I}$ & $\mathrm{Ec}$ & $\mathrm{Pc}$ \\
S. simplex & $\mathrm{S}, \mathrm{I}$ & $\mathrm{K}$ & \\
\hline
\end{tabular}

S, Solitary; G, Gregarious; I, Idiobiont; K, Koinobiont; Ec, Ectoparasitoid; En, Endoparasitoid; L, Larvae; L1, Larva fisrt instar; L2, Larva second instar; L3, Larva third instar; P, Pupae; U, Unknown.

\subsection{Introduced Parasitoids}

In the Americas and Hawaii, 29 species from 12 genera belonging to 7 families (Braconidae, Chalcididae, Diapriidae, Eulophidae, Figitidae, Ichneumonidae, and Pteromalidae) were introduced. Most introductions were species in the family Braconidae (72.4\%). Psyttalia are associated with all fruit flies of economic importance, Fopius, Dirhinus, Tetrastichus, and Pachycrepoideus are associated with three fruit fly genera, Coptera and Aceratoneuromyia with two genera, while Bathyplectes, Bracon, and Utetes with only one genus (Table 5).

Table 5. Association between genera of introduced parasitoids (Hymenoptera) with the genus of fruit flies with economic importance in the Americas and Hawaii.

\begin{tabular}{|c|c|c|c|c|c|}
\hline Parasitoid Species & Anastrepha & Bactrocera & Ceratitis & Rhagoletis & Zeugodacus \\
\hline \multicolumn{6}{|l|}{ Braconidae } \\
\hline Bracon & & $x$ & & & \\
\hline Diachasmimorpha & $\mathrm{X}$ & $X$ & $\mathbf{X}$ & & \\
\hline Fopius & $x$ & $x$ & $x$ & & \\
\hline Psyttalia & $X$ & $X$ & $x$ & $x$ & $x$ \\
\hline Utetes & & $x$ & & & \\
\hline \multicolumn{6}{|l|}{ Chalcididae } \\
\hline Dirhinus & $x$ & $x$ & $x$ & & \\
\hline \multicolumn{6}{|l|}{ Diapriidae } \\
\hline Coptera & & $x$ & $\mathbf{X}$ & & \\
\hline \multicolumn{6}{|l|}{ Eulophidae } \\
\hline Aceratoneuromyia & $X$ & & $X$ & & \\
\hline
\end{tabular}




\begin{tabular}{ccccc}
\hline Tetrastichus & $\mathrm{X}$ & $\mathrm{X}$ & $\mathrm{X}$ & \\
Figitidae & & & & \\
Aganaspis & $\mathbf{X}$ & & $\mathbf{X}$ & \\
Ichneumonidae & & & & $\mathrm{X}$ \\
Bathyplectes & & & & \\
Pteromalidae & & $\mathbf{X}$ & $\mathbf{X}$ & \\
Pachycrepoideus & $\mathbf{X}$ & $\mathrm{X}$ & \\
\hline
\end{tabular}

Diachasmimorpha longicaudata is the exotic parasitoid species with the most recorded hosts (12 species), and most widely distributed in the Americas. Diachasmimorpha tryoni and Fopius arisanus with 10 and 8 hosts, respectively, are also widely dispersed in the Americas (Table 6). The three braconid species previously mentioned are used against pestiferous tephritid species in Hawaii. Several introduced parasitoid species have been lab-reared, but until now they have not been released in the field, such as Bracon celer Szépligeti, Fopius caudatus (Szépligeti), Fopius ceratitivorus Wharton, Psyttalia ponerophaga (Silvestri), and Utetes africanus (Szépligeti), while other parasitoid species were released but did not establish, such as Psyttalia concolor (Szépligeti), Psyttalia cyclogaster (Thomson) (recorded previously as Coeloreuteus formosanus) (Watanabe) [69], Psyttalia humilis Silvestri, Psyttalia perproxima (Silvestri), and Aganaspis daci (Weld) (Table 6). Other parasitoid species were released but their establishment is unknown, either because there was no post-release follow-up, such as Psyttalia rhagoleticola (Sachtleben), Coptera silvestrii (Kieffer), and Bathyplectes exiguus Gravenhorst, or because of misidentifications with similar species that were collected from field surveys, such as Fopius persulcatus (Silvestri). Specimens belonging to Fopius vandenboschi (Fullaway) and Fopius arisanus (Sonan) recovered from field samples in Hawaii were mistakenly identified as F. persulcatus $[115,116]$.

Table 6. Introduced parasitoid species and their target tephritid fruit fly species in the Americas and Hawaii.

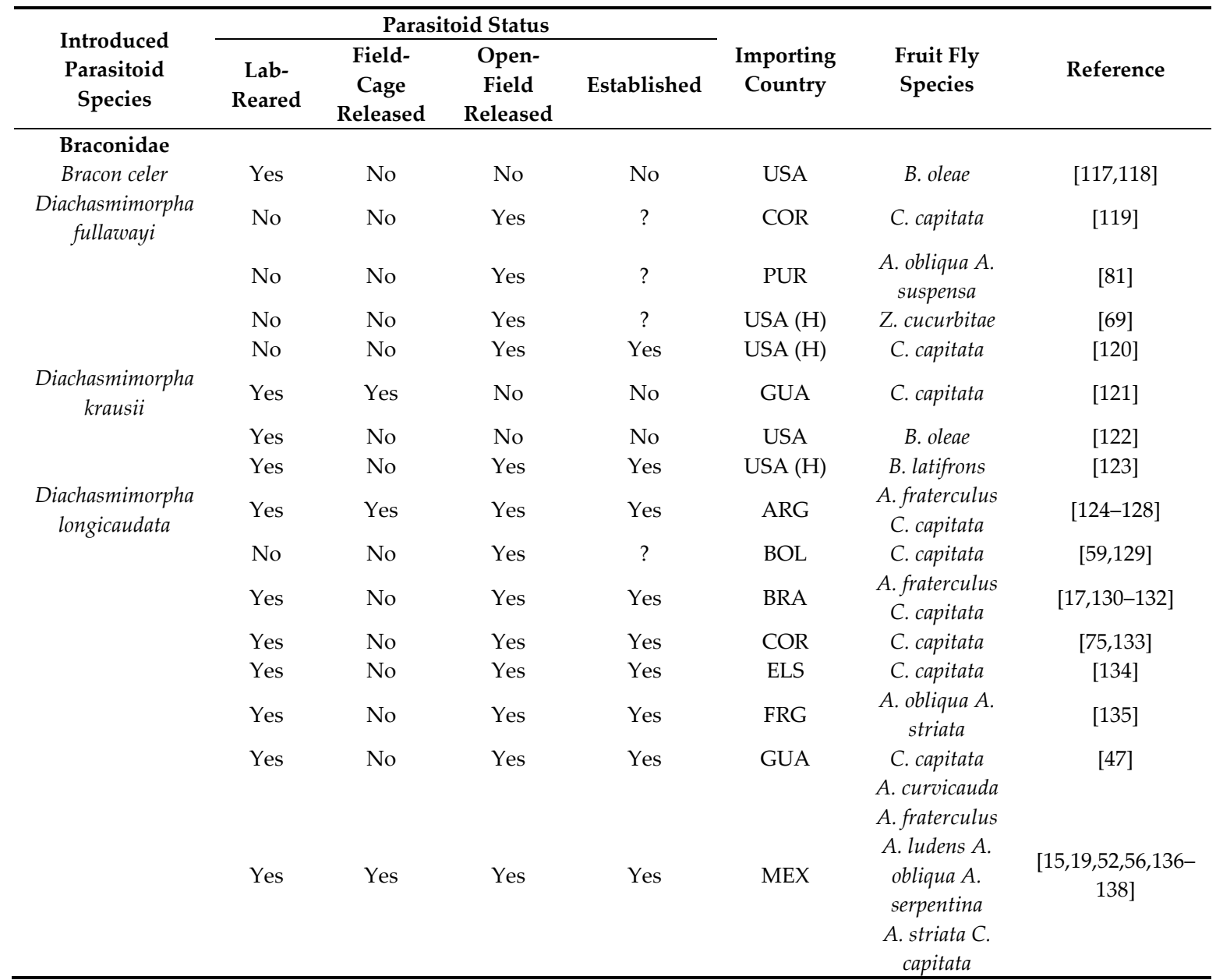




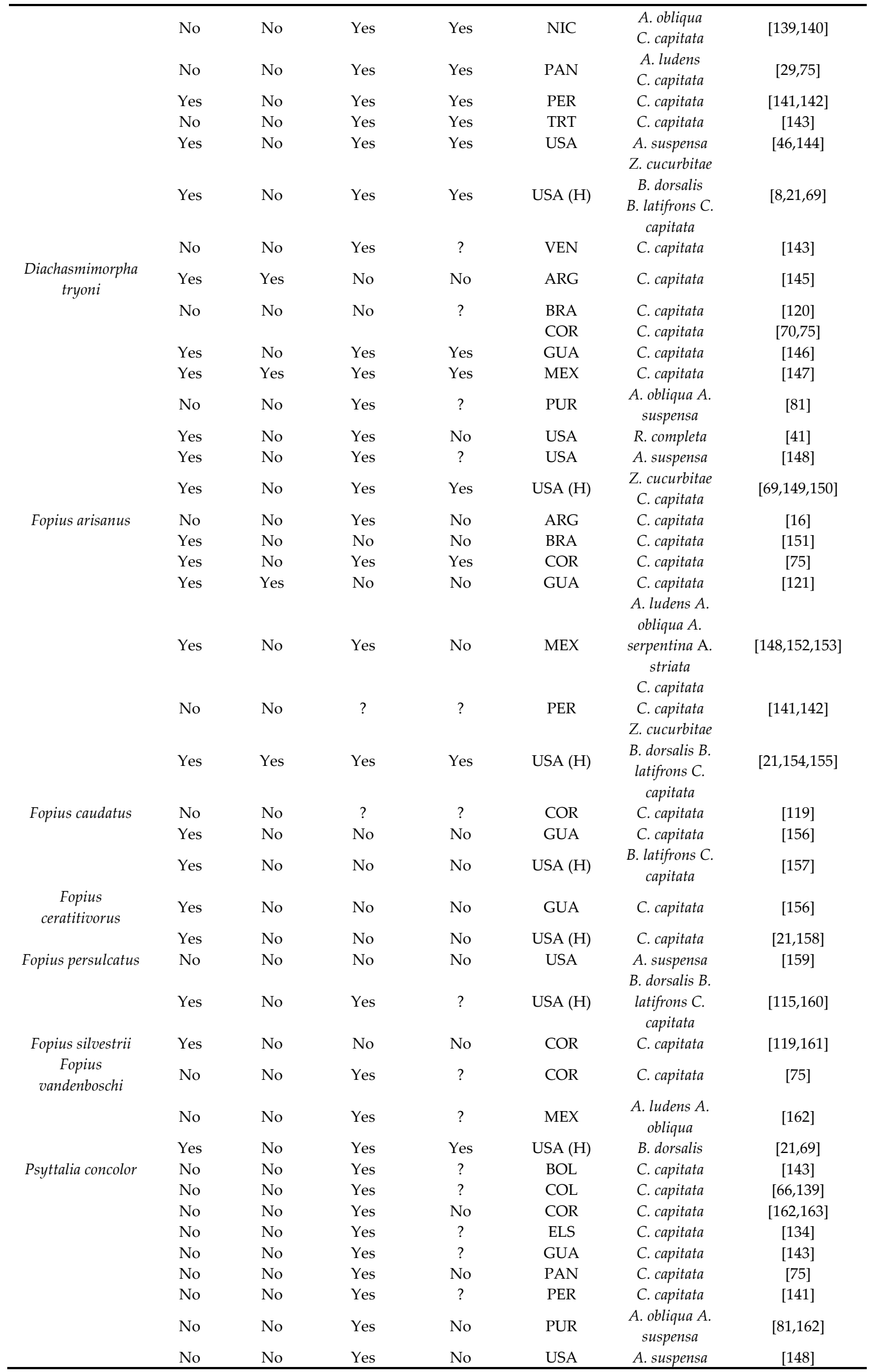




\begin{tabular}{|c|c|c|c|c|c|c|c|}
\hline $\begin{array}{c}\text { Psyttalia } \\
\text { cyclogaster }\end{array}$ & No & No & Yes & No & USA & Z. cucurbitae & [69] \\
\hline \multirow[t]{4}{*}{ Psyttalia fletcheri } & No & No & $?$ & $?$ & BRA & C. capitata & [120] \\
\hline & No & No & Yes & No & PUR & $\begin{array}{l}\text { A. obliqua A. } \\
\text { suspensa }\end{array}$ & {$[81,162]$} \\
\hline & No & No & Yes & No & USA & A. suspensa & [148] \\
\hline & Yes & Yes & Yes & Yes & USA (H) & $\begin{array}{l}\text { Z. cucurbitae } \\
\text { C. capitata }\end{array}$ & {$[8,21,155]$} \\
\hline \multirow[t]{2}{*}{ Psyttalia humilis } & Yes & No & Yes & No & USA $(\mathrm{H})$ & Z. cucurbitae & {$[69,164,165]$} \\
\hline & Yes & No & Yes & No & USA & B. oleae & {$[166,167]$} \\
\hline \multirow[t]{3}{*}{ Psyttalia incisi } & No & No & Yes & No & COR & C. capitata & {$[162,163]$} \\
\hline & No & No & Yes & No & MEX & $\begin{array}{l}\text { A. ludens A. } \\
\text { obliqua }\end{array}$ & {$[148,162]$} \\
\hline & Yes & No & Yes & Yes & USA (H) & $\begin{array}{l}\text { B. dorsalis } B \text {. } \\
\text { latifrons }\end{array}$ & [150] \\
\hline $\begin{array}{l}\text { Psyttalia } \\
\text { lounsburyi }\end{array}$ & Yes & No & Yes & Yes & USA & B. oleae & {$[167,168]$} \\
\hline $\begin{array}{c}\text { Psyttalia } \\
\text { perproxima }\end{array}$ & No & No & Yes & No & COR & C. capitata & [119] \\
\hline $\begin{array}{c}\text { Psyttalia } \\
\text { ponerophaga }\end{array}$ & Yes & No & No & No & USA & B. oleae & [169] \\
\hline $\begin{array}{c}\text { Psyttalia } \\
\text { rhagoleticola }\end{array}$ & No & No & Yes & $?$ & CAN & R. pomonella & [112] \\
\hline $\begin{array}{l}\text { Utetes africanus } \\
\text { Chalcididae }\end{array}$ & Yes & No & No & No & USA & B. oleae & {$[167,170]$} \\
\hline Dirhinus anthracia & Yes & No & Yes & Yes & USA (H) & Z. cucurbitae & {$[69,150]$} \\
\hline \multirow{7}{*}{ Dirhinus giffardii } & No & No & Yes & ? & BOL & C. capitata & {$[171]$} \\
\hline & No & No & Yes & $?$ & COL & C. capitata & [139] \\
\hline & No & No & Yes & $?$ & COR & C. capitata & [163] \\
\hline & No & No & Yes & $?$ & MEX & $\begin{array}{l}\text { A. ludens } A \text {. } \\
\text { obliqua }\end{array}$ & [148] \\
\hline & No & No & Yes & $?$ & PER & C. capitata & [141] \\
\hline & No & No & Yes & No & USA & A. suspensa & [148] \\
\hline & Yes & No & Yes & Yes & USA $(\mathrm{H})$ & $\begin{array}{l}\text { Z. cucurbitae } \\
\text { C. capitata }\end{array}$ & {$[69,172]$} \\
\hline \multicolumn{8}{|l|}{ Diapriidae } \\
\hline Coptera silvestrii & Yes & No & Yes & $?$ & USA $(\mathrm{H})$ & $\begin{array}{l}\text { Z. cucurbitae } \\
\text { C. capitata }\end{array}$ & {$[69,120]$} \\
\hline \multicolumn{8}{|l|}{ Eulophidae } \\
\hline \multirow[t]{12}{*}{$\begin{array}{l}\text { Aceratoneuromyia } \\
\text { indica }\end{array}$} & Yes & No & Yes & Yes & ARG & $\begin{array}{l}\text { A. fraterculus } \\
\text { C. capitata }\end{array}$ & [86] \\
\hline & No & No & Yes & Yes & BOL & $\begin{array}{l}\text { C. capitata } \\
\text { A. fraterculus }\end{array}$ & {$[59,139]$} \\
\hline & No & No & Yes & Yes & $\mathrm{COL}$ & $\begin{array}{l}\text { A. striata } C . \\
\text { capitata }\end{array}$ & {$[66,139]$} \\
\hline & No & No & Yes & $?$ & COR & C. capitata & [75] \\
\hline & No & No & Yes & $?$ & GUA & C. capitata & [173] \\
\hline & No & No & Yes & Yes & MEX & $\begin{array}{l}\text { A. ludens A. } \\
\text { obliqua }\end{array}$ & {$[52,174]$} \\
\hline & No & No & Yes & Yes & NIC & C. capitata & [75] \\
\hline & No & No & Yes & $?$ & PAN & C. capitata & [75] \\
\hline & No & No & $?$ & $?$ & PER & C. capitata & [141] \\
\hline & No & No & ? & ? & TRT & C. capitata & [143] \\
\hline & No & No & Yes & Yes & USA (H) & C. capitata & [120] \\
\hline & No & No & Yes & Yes & VEN & C. capitata & [143] \\
\hline \multirow{6}{*}{$\begin{array}{l}\text { Tetrastichus } \\
\text { giffardianus }\end{array}$} & No & No & Yes & No & ARG & C. capitata & [16] \\
\hline & Yes & No & Yes & Yes & BRA & C. capitata & {$[120,175]$} \\
\hline & No & No & Yes & $?$ & PER & C. capitata & [141] \\
\hline & No & No & Yes & No & PUR & $\begin{array}{l}\text { A. obliqua A. } \\
\text { suspensa }\end{array}$ & [81] \\
\hline & No & No & Yes & $?$ & USA & A. suspensa & [148] \\
\hline & Yes & No & Yes & Yes & USA (H) & $\begin{array}{l}\text { Z. cucurbitae } \\
\text { B. latifrons }\end{array}$ & {$[21,69]$} \\
\hline
\end{tabular}




\begin{tabular}{|c|c|c|c|c|c|c|c|}
\hline \multirow[t]{5}{*}{ Aganaspis daci } & No & No & Yes & $?$ & COR & C. capitata & {$[163]$} \\
\hline & No & No & Yes & $?$ & COL & C. capitata & [139] \\
\hline & No & No & Yes & $?$ & MEX & $\begin{array}{c}\text { A. ludens } A \text {. } \\
\text { obliqua }\end{array}$ & [176] \\
\hline & No & No & Yes & No & USA & A. suspensa & [159] \\
\hline & No & No & Yes & $?$ & USA $(\mathrm{H})$ & C. capitata & [120] \\
\hline \multicolumn{8}{|l|}{ Ichneumonidae } \\
\hline $\begin{array}{l}\text { Bathyplectes } \\
\text { exiguus }\end{array}$ & No & No & Yes & $?$ & CAN & R. meiiginii & [112] \\
\hline \multirow[t]{5}{*}{$\begin{array}{c}\text { Pteromalidae } \\
\text { Pachycrepoideus } \\
\text { vindemmiae }\end{array}$} & Yes & No & Yes & Yes & ARG & $\begin{array}{l}\text { A. fraterculus, } \\
\text { C. capitata }\end{array}$ & [16] \\
\hline & Yes & No & Yes & Yes & BRA & A. fraterculus & [177] \\
\hline & Yes & No & Yes & Yes & COR & C. capitata & [163] \\
\hline & Yes & No & Yes & Yes & MEX & A. serpentina & [52] \\
\hline & Yes & No & Yes & Yes & USA (H) & $\begin{array}{l}\text { Z. cucurbitae } \\
\text { C. capitata }\end{array}$ & {$[69,172]$} \\
\hline
\end{tabular}

Countries: ARG, Argentina; BEL, Belize; BOL, Bolivia; BRA, Brazil; CAN, Canada;, COL, Colombia; COR, Costa Rica; CUB, Cuba; DOR, Dominican Republica; ECU, Ecuador; ELS, El Salvador; FRG, French Guiana; GUA, Guatemala; MEX, Mexico; NIC, Nicaragua; PAN, Panama; PER, Peru; PUR, Puerto Rico; TRT, Trinidad and Tobago; VEN, Venezuela; USA (C), United States of America, California; USA (F), United States of America; USA (H), United States of America, Hawaii.

Most of the parasitoids introduced are solitary (89.6\%), koinobiontes, (82.8\%), and endoparasitoids $(86.2 \%)$. Only 3 species present a gregarious habit and are restricted to the Eulophidae and Pteromalidae families. The larval stage is the most attacked stage (75.9\%), followed by the pupa (13.8\%) and egg (10.3\%). Only Dirhinus anthracia Walker, Dirhinus giffardii Silvestri, and Pachycrepoideus vindemmiae (Rondani) are ectoparasitoids and idiobionts, while Coptera silvestrii is an endoparasitoid and idiobiont parasitoid (Table 7).

Table 7. Guilds of introduced parasitoids of tephritid fruit flies in the Americas and Hawaii.

\begin{tabular}{cccc}
\hline Introduced Parasitoid Species & Parasitism Modes & Feeding Types & Host Stage Attacked \\
\hline Braconidae & & & \\
Bracon celer & S, K & En & L3 \\
Diachasmimorpha fullawayi & S, K & En & L3 \\
D. krausii & S, K & En & L2-L3 \\
D. longicaudata & S, K & En & L2-L3 \\
D. tryoni & S, K & En & L3 \\
Fopius ceratitivorus & S, K & En & E-L1 \\
F. caudatus & S, K & En & E \\
F. arisanus & S, K & En & E \\
F. persulcatus & S, K & En & L1 \\
F. silvestrii & S, K & En & L2-L3 \\
F. vandenboschi & S, K & En & L1 \\
Psyttalia concolor & S, K & En & L2-L3 \\
P. cyclogaster & S, K & En & L? \\
P. fletcheri & S, K & En & L3 \\
P. humilis & S, K & En & L3 \\
P. incisi & S, K & En & L3 \\
P. lounsburyi & S, K & En & \\
P. perproxima & S, K & En & L2-L3 \\
P. ponerophaga & S, K & En & L2-L3 \\
P. rhagoleticola & S, K & En & L2-L3 \\
Utetes africanus & S, K & En & L2-L3 \\
Chalcididae & & & \\
Dirhinus anthracia & S, I & Ec & P
\end{tabular}




\begin{tabular}{|c|c|c|c|}
\hline D. giffardii & $S, I$ & Ec & $\mathrm{P}$ \\
\hline \multicolumn{4}{|l|}{ Diapriidae } \\
\hline Coptera silvestrii & $S, I$ & En & $\mathrm{P}$ \\
\hline \multicolumn{4}{|l|}{ Eulophidae } \\
\hline Aceratoneuromyia indica & $\mathrm{G}, \mathrm{K}$ & En & L3 \\
\hline Tetrastichus giffardianus & $\mathrm{G}, \mathrm{K}$ & En & L3 \\
\hline \multicolumn{4}{|l|}{ Figitidae } \\
\hline Aganaspis daci & S, K & En & L2-L3 \\
\hline \multicolumn{4}{|l|}{ Ichneumonidae } \\
\hline Bathyplectes exiguus & $\mathrm{U}$ & $\mathrm{U}$ & $\mathrm{L}$ \\
\hline \multicolumn{4}{|l|}{ Pteromalidae } \\
\hline Pachycrepoideus vindemmiae & S-G, I & Ec & $\mathrm{P}$ \\
\hline
\end{tabular}

S, Solitary; G, Gregarious; I, Idiobiont; K, Koinobiont; Ec, Ecotoparasitoid; En, Endoparasitoid; L, Lavae; L1, Lava fisrt instar; L2, Larva second instar; L3, Larva third instar; P, Pupae; U, Unknown.

\section{Predators}

A total of 56 species of fruit fly predators occur in the Americas and Hawaii, including 11 spiders (Araneae: Araneidae, Lycosidae, Philodromidae, and Salticidae), 2 mites (Acari: Macrochelidae), 3 crickets (Orthoptera: Gryllidae and Trigonidiidae), 14 beetles (Coleoptera: Carabidae, Elateridae, and Stathyllinidae), 17 ants (Hymenoptera: Formicidae), 2 earwig (Dermaptera: Anisolabididae and Chelisochidae), 1 true bug (Reduviidae), 1 lizard (Reptilia: Polychrotidae), and 2 rodents (Rodentia: Vespertilionidae) (Table 8). From these predators, only the prey of spiders on the adult phase of fruit flies, the others prey on larvae and/pupae in the soil.

The genus Anastrepha has 28 predators associated ( 9 with A. ludens, 8 with A. suspensa, 6 with A. fraterculus, and 5 with unidentified Anastrepha species), followed by Rhagoletis with 21 (16 species with $R$. pomonella and 5 with $R$. mendax), and Ceratitis capitata with 4 . There are no predators of Bactrocera in the Americas.

Only 6 countries (Brazil, Canada, Cuba, Guatemala, Mexico, and USA) have a record of the economic importance fruit fly predators in the Americas. Canada has a richness of 20 species of predators, followed by USA (15 spp.), Brazil (11 spp.), Mexico (3 spp.), Cuba (1 sp.), and Guatemala (1 sp.) (Table 8).

Table 8. Predators of fruit flies in the Americas and Hawaii.

\begin{tabular}{|c|c|c|c|c|c|}
\hline Predator & $\mathbf{O}$ & Ps & Prey & $\mathrm{C}$ & Reference \\
\hline \multicolumn{6}{|l|}{ Araneae } \\
\hline \multicolumn{6}{|l|}{ Araneidae } \\
\hline Argiope sp. & $\mathbf{N}$ & $\mathrm{A}$ & Z. curcubitae & USA $(\mathrm{H})$ & {$[178,179]$} \\
\hline \multicolumn{6}{|l|}{ Lycosidae } \\
\hline Trochosa terricola & $\mathrm{N}$ & $\mathrm{A}$ & R. pomonella & CAN & [180] \\
\hline \multicolumn{6}{|l|}{ Philodromidae } \\
\hline Philodrornus praelustris & $\mathrm{N}$ & $\mathrm{A}$ & R. pomonella & CAN & [181] \\
\hline Philodromus vulgaris & $\mathrm{N}$ & A & R. pomonella & CAN & [181] \\
\hline \multicolumn{6}{|l|}{ Salticidae } \\
\hline Eris militaris & $\mathrm{N}$ & $\mathrm{A}$ & R. pomonella & CAN & {$[181]$} \\
\hline Megafreya sutrix & $\mathrm{N}$ & A & A. fraterculus & BRA & {$[182,183]$} \\
\hline Paraphidippus aurantius & $\mathrm{N}$ & $\mathrm{A}$ & A. ludens & MEX & {$[184]$} \\
\hline Pelegrina proterva & $\mathrm{N}$ & A & R. pomonella & CAN & {$[181]$} \\
\hline Phidippus audax & $\mathbf{N}$ & $\mathrm{A}$ & A. ludens & MEX & [185] \\
\hline Phidippus bidentatus & $\mathbf{N}$ & A & A. ludens & MEX & {$[184]$} \\
\hline Salticus scenicus & $\mathrm{N}$ & $\mathrm{A}$ & Rhagoletis sp. & CAN & [69] \\
\hline \multicolumn{6}{|l|}{ Acari: Macrochelidae } \\
\hline Macrocheles sp. & $\mathrm{N}$ & A & A. suspensa & CUB & [186] \\
\hline Macrocheles roquensis & $\mathrm{N}$ & $\mathrm{A}$ & C. capitata & BRA & [187] \\
\hline
\end{tabular}




\begin{tabular}{|c|c|c|c|c|c|}
\hline \multicolumn{6}{|l|}{ Gryllidae } \\
\hline Gryllus pennsylvanicus & $\mathrm{N}$ & L.P & R. pomonella & CAN & {$[180,188]$} \\
\hline \multicolumn{6}{|l|}{ Trigonidiidae } \\
\hline \multicolumn{6}{|l|}{ Dermaptera } \\
\hline \multicolumn{6}{|l|}{ Anisolabididae } \\
\hline \multicolumn{6}{|l|}{ Chelisochidae } \\
\hline $\begin{array}{c}\text { Chelisoches morio } \\
\text { Coleoptera } \\
\text { Carabidae }\end{array}$ & $\mathbf{E}$ & $\mathrm{L}$ & Z. curcubitae & USA $(\mathrm{H})$ & {$[178,179]$} \\
\hline Amara aenea & $\mathrm{N}$ & $\mathrm{L}, \mathrm{P}$ & R. pomonella & CAN & [180] \\
\hline Calosoma calidum & $\mathrm{N}$ & L.P & R. pomonella & CAN & [180] \\
\hline Carabus nemoralis & $\mathrm{N}$ & $\mathrm{L}$ & R. mendax & CAN & [190] \\
\hline Harpalus aeneus & $\mathrm{N}$ & $\mathrm{L}, \mathrm{P}$ & R. pomonella & CAN & [180] \\
\hline Harpalus pennsylvanicus & $\mathrm{N}$ & L.P & R. pomonella & CAN & [180] \\
\hline Poecilus lucublandus & $\mathrm{N}$ & $\mathrm{L}$ & R. mendax & CAN & [190] \\
\hline \multirow[t]{2}{*}{ Pterostichus melanarius } & $\mathrm{E}$ & $\mathrm{L}, \mathrm{P}$ & R. pomonella & CAN & {$[180]$} \\
\hline & & $\mathrm{L}, \mathrm{P}$ & R. mendax & CAN & [191] \\
\hline \multicolumn{6}{|l|}{ Elateridae } \\
\hline \multicolumn{6}{|l|}{ Staphylinidae } \\
\hline \multirow[t]{3}{*}{ Belonochus rufipennis } & $\mathrm{N}$ & $\mathrm{L}$ & A. fraterculus & BRA & [192] \\
\hline & & $\mathrm{L}$ & A. ludens & MEX & [193] \\
\hline & & $\mathrm{L}$ & C. capitata & BRA & [192] \\
\hline Dinothenarus badipes & $\mathrm{N}$ & $\mathrm{L}, \mathrm{P}$ & R. pomonella & CAN & [180] \\
\hline Homaetarsus sp. & $\mathrm{N}$ & $\mathrm{L}$ & A. ludens & MEX & [194] \\
\hline Philonthus sp. & $\mathrm{N}$ & $\mathrm{L}, \mathrm{P}$ & R. pomonella & CAN & [180] \\
\hline Philonthus turbidus & $\mathrm{E}$ & $\mathrm{L}$ & Z. curcubitae & USA (H) & {$[178,179]$} \\
\hline \multicolumn{6}{|l|}{ Hemiptera: Reduviidae } \\
\hline Zelus renardii & $\mathrm{E}$ & A & Z. curcubitae & USA (H) & {$[178,179]$} \\
\hline \multicolumn{6}{|l|}{ Hymenoptera: Formicidae } \\
\hline Crematogaster sp. & $\mathrm{N}$ & $\mathrm{L}$ & A. ludens & USA & [194] \\
\hline Dorymyrmex sp. 1 & $\mathrm{~N}$ & $\mathrm{~L}$ & Anastrepha sp & BRA & {$[23]$} \\
\hline Ectatomma brunneum & & $\mathrm{L}$ & Anastrepha sp & BRA & [23] \\
\hline Formica fusca & $\mathrm{N}$ & $\mathrm{L}, \mathrm{P}$ & R. pomonella & CAN & {$[180]$} \\
\hline Leptothorax sp. & $\mathrm{N}$ & $\mathrm{L}, \mathrm{P}$ & A. suspensa & USA & [189] \\
\hline Monomorium sp. & $\mathrm{N}$ & $\mathrm{L}, \mathrm{P}$ & A. suspensa & USA & [189] \\
\hline Myrmica sp. & $\mathrm{N}$ & $\mathrm{L}, \mathrm{P}$ & R. pomonella & CAN & {$[180]$} \\
\hline Pachycondyla striata & $\mathrm{N}$ & $\mathrm{L}$ & A. fraterculus & BRA & {$[25]$} \\
\hline Paratrechina paroula & $\mathrm{N}$ & $\mathrm{L}, \mathrm{P}$ & A. suspensa & USA & [189] \\
\hline \multirow[t]{2}{*}{ Pheidole gertrude } & $\mathrm{N}$ & $\mathrm{L}$ & Anastrepha sp & $\mathrm{BR}$ & {$[23]$} \\
\hline & $\mathrm{N}$ & $\mathrm{L}$ & A. ludens & MEX & [193] \\
\hline Pheidole magacephala & $\mathrm{E}$ & E, L, P & Z. curcubitae & USA $(\mathrm{H})$ & {$[178,179]$} \\
\hline Pheidole oxyops & $\mathrm{N}$ & $\mathrm{L}$ & Anastrepha sp & BRA & {$[23]$} \\
\hline Pogonomyrmex naegelli & $\mathrm{N}$ & $\mathrm{L}$ & A. fraterculus & BRA & {$[25]$} \\
\hline Odontomachus brunneus & $\mathrm{E}$ & $\mathrm{L}, \mathrm{P}$ & A. suspensa & USA & [189] \\
\hline \multirow[t]{5}{*}{ Solenopsis geminata } & $\mathrm{N}$ & $\mathrm{L}$ & A. ludens & MEX & {$[22,193]$} \\
\hline & $\mathrm{E}$ & $\mathrm{L}$ & A. ludens & USA & {$[194]$} \\
\hline & $\mathrm{E}$ & E, L, P & Z. curcubitae & USA $(\mathrm{H})$ & {$[178,179]$} \\
\hline & $\mathrm{N}$ & $\mathrm{L}$ & C. capitata & GUA & [195] \\
\hline & $\mathrm{E}$ & $\mathrm{P}$ & C. capitata & USA & [69] \\
\hline Solenopsis invicta & $\mathrm{E}$ & $\mathrm{L}$ & A. suspensa & USA & [189] \\
\hline Solenopsis saevissima & $\mathrm{N}$ & $\mathrm{L}$ & A. fraterculus & BRA & [25] \\
\hline \multicolumn{6}{|l|}{ Reptilia: Polychrotidae } \\
\hline Anolis serranoi & $\mathrm{N}$ & $\mathrm{L}$ & A. ludens & MEX & [196] \\
\hline
\end{tabular}




\section{Rodentia: Cricetidae}

Peromyscus leucopus

Peromyscus boylii

\begin{tabular}{rrrrr}
$\mathrm{N}$ & $\mathrm{P}$ & A. ludens & MEX & {$[197]$} \\
$\mathrm{N}$ & $\mathrm{P}$ & A. ludens & MEX & {$[197]$} \\
\hline
\end{tabular}

O, Origin; N, Native; E, Exotic; C, Country; PS, Pray stage attached PS; A, Adult; E, Egg; L, Larvae; P, Pupae; BRA, Brazil; CAN, Canada; CUB, Cuba; GUA, Guatemala; MEX, Mexico; USA, United States of America; USA (H), United States of America, Hawaii.

Ants are a group of predatory insects that can be considered as pest control agents in some agroecosystems, regulating insect populations [198]. The predation of fruit flies by ants occurs when the larva leaves the fruit to bury itself in the soil for pupation [23]. Ants belonging to the genus Pachycondyla, Pheidole, Pogonomyrmex, and Solenopsis are important predators of $A$. fraterculus larvae in Brazil. Solenopsis saevissima was the most efficient species, with $42.86 \%$ of larvae removal in the field [25].

\section{Biological Control Programs}

Introduction of the invasive Zeugodacus cucurbitae (melon fly), Bactocera dorsalis (Hendel) (oriental fruit fly), and Bactrocera latifrons (Hendel) into Hawaii resulted initially in classical biological control programs, but later they became augmentative biological control programs $[8,10,160,164]$. Numerous parasitoid and predator species were introduced into Hawaii for classical biological control of Bactrocera spp. $[69,178,179]$. However, only the Asian-native larval parasitoid Psyttalia fletcheri (Silvestri) was successfully established in Hawaii on Z. cucurbitae with parasitism percentages that varied according to the host fruit species [8]. In an augmentative release program against the melon fly, $P$. fletcheri substantially reduced the number of emerged flies $[155,199]$. Other exotic parasitoid species, from Southern Asia and other regions, were successfully established on B. dorsalis in Hawaii, such as larval parasitoids Diachasmimorpha longicaudata (Ashmead), Psyttalia incisi (Silvestri), Fopius vandenboschi (Fullaway), and Tetrastichus giffardianus Silvestri, the egg-larval parasitoid Fopius arisanus (Sonan) and the pupal parasitoids Dirhinus giffardii (Silvestri) and Pachycrepoideus vindemmiae Rondani [154,200]. Augmentative releases of D. longicaudata made against B. dorsalis were inconsistent because they produced lower fly populations in the release plots one year and higher populations the next [10]. Although both $D$. longicaudata and $F$. vandenboschi were important biological control agents of $B$. dorsalis, $F$. arisanus has remained the most significant parasitoid of this tephritid species [8]. Because of the F. arisanus habit of attacking host eggs, which are more exposed below the fruit skin surface than larvae, this braconid parasitoid can achieve host parasitism percentages between $60 \%$ and $70 \%$ in the field [10]. In addition, F. arisanus was also the predominant species recovered from B. latifrons [158].

The establishment of the olive fruit fly B. oleae in California, USA, where it has spread to all commercial olive-producing areas since first being detected in 1998 [201], led to the development of a classical biological program control program in 2003 [167]. Several parasitoid species recovered from B. oleae collected from wild olives in Kenya, South Africa, Pakistan, or Namibia were imported to the USA. The introduced species were Bracon celer Szépligeti, Psyttalia humilis (Silvestri) (P. humilis from Kenya was previously referred to as P. cf. concolor) [165,202], P. lounsburyi (Silvestri), $P$. ponerophaga (Silvestri), and Utetes africanus (Silvestri) [117,118,165,168-170]. In addition, three exotic parasitoid species, i.e., the Australian-native Fopius arisanus, Diachasmimorpha kraussii (Fullaway), and $D$. longicaudata, coming from colonies in Hawaii, were also evaluated as potential biological control agents for B. oleae [122,203]. Although both D. longicaudata and D. kraussii were efficient against $B$. oleae [122], they were not considered for field releases because both braconid species are hostgeneralists. Given this, more specialized species such as the larval parasitoids $P$. humilis and $P$. lounsburyi were chosen to release in California [168,204]. Field release and recovery efforts were conducted from 2006 to 2013; both parasitoid species were recovered post-release, but only $P$. lounsburyi was established in California coastal regions [167,205]. Given these results, the parasitoid P. lounsburyi was mass-reared for release on a larger scale in olive-producing areas of California [206].

An augmentative biological control program against the introduced B. carambolae, the carambola fruit fly, was carried out in Northern Brazil (Amapá state) by releasing millions of D. longicaudata 
specimens [151]. Although D. longicaudata adapted to the Amazonian environment [17], it did not have a substantial effect in controlling the tephritid target. The Asian-native parasitoid D. longicaudata was previously introduced in 1994 into Brazil from Gainesville, Florida, United States, for use against C. capitata and Anastrepha spp. [17]. In 2012, a new biological control program against B. carambolae was started by introducing F. arisanus into Brazil from Hawaii; currently, this braconid parasitoid is reared on C. capitata eggs in different Embrapa laboratories and in the Moscamed Brazil facility [151].

The first classical biological control programs against $C$. capitata generally involved the introduction of parasitoid and predator species not only for the control of this pest but also for using them against other pestiferous tephritid species, such as Bactrocera spp. [21] or Anastrepha spp. [11]. The earliest classical biological control programs against $C$. capitata were carried out in Hawaii and date as far back as 1913. Because of these biocontrol programs, several parasitoid species, such as the larval parasitoids Aceratoneuromyia indica (Silvestri), Diachasmimorpha fullawayi (Silvestri), D. tryoni (Cameron), D. longicaudata, F. vandenboschi, P. incisi, Aganaspis daci (Weld), Tetrastichus giffardianus Silvestri, the egg-larval parasitoid $F$. arisanus, the pupal parasitoids Coptera silvestrii (Kieffer), Dirhinus giffardii Silvestri, and P. vindemmiae, were introduced to Hawaii and most of those parasitoid species were successfully established on C. capitata [120]. However, the Asian-native F. arisanus, since its establishment in the late 1940s, became the major parasitoid of $C$. capitata through substantial reductions in the medfly population in some habitats, apart from controlling $B$. dorsalis $[8,115,207]$. In spite of this, the implementation of classic biological control programs in Hawaii did not meet the objectives expected for the control of pestiferous fruit fly species, which motivated the development of mass-rearing of different parasitoid species for their periodic augmentative release in the field [208-210]. Therefore, using augmentative releases of parasitoids as a strategy into integrated management programs to control C. capitata, as well as for other pestiferous fruit fly species, has been encouraged since the 1980s $[149,154,211]$. Thus, augmentative releases of $D$. tryoni were performed in the late 1980s in Hawaii, due to its simplicity for mass-rearing and its host preference for $C$. capitata rather than B. dorsalis [149]. Those augmentative releases of $D$. tryoni were able to suppress medfly populations and the combination with sterile male fly releases had a greater effect on the pest [212]. However, high medfly populations still occur in Hawaii mainly in coffee plantations and at higher elevations [213]. Newly classical biological control programs carried out against C. capitata in Hawaii focused on the introduction of more specific parasitoid species [214,215]. New introduced species were the larval parasitoid D. kraussii and the Eastern African-native egg-pupal parasitoids Fopius ceratitivorus Wharton and F. caudatus (Szépligeti) [213]. Among these parasitoid species, F. ceratitivorus would be the most promising for improving overall suppression of medfly in Hawaii, due to its host specificity, lack of non-target impacts, and ability to complement $F$. arisanus $[158,213,216,217]$.

Since the establishment of $C$. capitata in Hawaii, this pestiferous fruit fly species has been periodically introduced and erradicated in California, Florida, and Texas (USA) as well in Southern Mexico, although high medfly populations still remain throughout Central and South America [121]. The northward spread of C. capitata from Central America into Mexico, and also into the United States, has been constantly monitored along the Mexican/Guatemalan border by the international organization Mosca del Mediterráneo (MOSCAMED) (United States, Mexico, and Guatemala) [121]. Predominantly in this region, the vast areas cultivated with coffee, Coffea arabica L., which extend through the highlands of Guatemala, maintain high medfly populations. In addition to the use of the Sterile Insect Technique (SIT) to control medfly populations, augmentative parasitoid releases have also been carried out. Diachasmimorpha tryoni has been augmentatively released from the air into coffee cultivated areas affected by C. capitata in Guatemala over two years, which led to parasitism levels of up to $84 \%$ [146]. Previously, Cancino et al. [218] showed the significant effects of $D$. longicaudata mass-releases on C. capitata populations infesting coffee berries on the MexicoGuatemala border. Similarly, augmentative releases of the Asian-native parasitoid D. longicaudata against medfly were carried out in Chiapas, Southern Mexico, during 2001 and 2002 on over 9000 ha of coffee plantations, reaching parasitism peaks of $61 \%$ and $69 \%$, respectively [138]. In addition, augmentative releases of $D$. krausii and F. arisanus, either together or in combination with medfly 
sterile males, were made inside large field cages erected over coffee grown at different locations and altitudes in Guatemala. Results showed that the inclusion of both parasitoid species provided significant medfly suppression and the effect was frequently substantial [121]. These outcomes indicate that augmentative releases of parasitoids could be a complementary tool to control high medfly populations within an area-wide integrated fruit fly management (AWIFFM) approach [219]. The introduction from Kenya of the medfly-specific parasitoid F. ceratitivorus to Guatemala, and its establishment in the USDA-APHIS/MOSCAMED quarantine facility at San Miguel Petapa, points to a new process to strengthen the use of parasitoids against these medflies [156]. Considering differences in weather conditions and medfly density throughout the area of the Mexican/Guatemalan border, several parasitoid species with different bioecological features have been reared in Guatemala by the MOSCAMED Program [121]. Not all parasitoid species are equally effective under all likely conditions; preferences for temperature, moisture, and/or host density may vary $[76,78]$.

Costa Rica was the first Central American country to develop a biological control program against C. capitata by introducing numerous parasitoid species mainly from Hawaii in the 1950s. Thus, F. arisanus, D. longicaudata, A. indica, $P$. concolor, and $P$. vindemmiae were released and recovered in Costa Rica, but the impact on C. capitata was not significant [11]. In the 1980s, a classic biological control program facilitated the introduction to Costa Rica of four parasitoid species from Africa, i.e., Diachasmimorpha fullawayi, Psyttalia perproxima (Silvestri) (recorded as P. perproximus (Silvestri)) [119], Fopius caudatus, and F. silvestrii (Wharton); this last species was previously misidentified as F. caudatus [220]. The four braconid parasitoid species were directly released in the field but there was no recovery of them post-release [119]. Currently, both D. longicaudata and P. vindemmiae are mass-reared in Costa Rica for fruit fly biological control, although little information is available on their present status parasitizing C. capitata [133]. The fruit fly biological control program developed by Costa Rica in the 1950s was essential for promoting the use of parasitoids in other Latin American countries affected by the medfly. Thus, $D$. longicaudata, $A$. indica, and $P$. vindemmiae were mainly provided by Costa Rica to Nicaragua, Panamá, El Salvador, Guatemala, Trinidad and Tobago (Central America), Argentina, Bolivia, Colombia, Perú, and Venezuela (South America).

The larval parasitoid T. giffardianus was the first exotic species introduced into both Brazil and Argentina during the 30s and 40s, respectively, for C. capitata control. Low numbers of individuals were released in both countries. In Brazil, this eulophid parasitoid was recovered from medfly puparia after 60 years from its first release [17], but in Argentina there has been no evidence of its permanent establishment at any release site [16]. New biological control programs that involved the introduction of several exotic parasitoid species into Argentina from Mexico and Costa Rica were carried out between the 1960s and 1990s. The establishment on C. capitata of three released parasitoid species, D. longicaudata, A. indica, and P. vindemmiae, was verified in Argentina, although without exercising significant control on this tephritid pest [16]. However, open-field augmentative releases of $D$. longicaudata mass-reared on irradiated larvae of the tsl Vienna-8 medfly strain (named as " $D$. longicaudata tsl-Cc line") have recently been carried out in fruit-growing areas of Central-Western Argentina. Post-release data showed up to $75 \%$ of $C$. capitata mortality due to the D. longicaudata releases in fig crops $[126,127]$. Later, augmentative releases of the D. longicaudata tsl-Cc line were carried out to assess the effectiveness of parasitoid females in killing medfly larvae infesting peach and orange inside a field cage in the subtropical environment of the northwestern Argentina. Parasitoid effectiveness reached up to $50 \%$ in infested peaches [128]. Recent studies on the massrearing of the neotropical pupal parasitoid Coptera haywardii (Ogloblin) using gamma-irradiated larvae of the tsl Vienna-8 medfly strain as the host have been carried out at the BioPlanta San Juan facility [20]. Furthermore, the possibility of augmentative releases of $C$. haywardii for medfly control is currently being evaluated in Argentina. Augmentative releases of $D$. longicaudata against $C$. capitata were also carried out in different Brazilian regions, but recoveries of this braconid parasitoid were more associated with Anastrepha spp. [17,131,132]. This braconid parasitoid species has been previously introduced in 1994 into Brazil from Gainesville, Florida, United States, for use against $C$. capitata and Anastrepha spp. [151]. 
Historically, the introduction and release of exotic hymenopterous parasitoid species for biological control of native pestiferous Anastrepha species have been mainly standardized from the 1930s in Puerto Rico, Costa Rica, and Mexico, as well as in many Latin American countries. Most of these parasitoid species had first been introduced into Hawaii including D. longicaudata, D. fullawayi, D. tryoni, P. incisi, P. concolor, P. fletcheri, F. arisanus, F. vandenboschi, A. daci, T. giffardianus, D. giffardii, A. indica, and $P$. vindemmiae. A few species were able to establish in the released areas and they were able to control the target Anastrepha species. The status of all those introduced parasitoid species was discussed by Ovruski et al. [11].

Since 1992, Mexico has carried out the main biological control program against pestiferous Anastrepha species in the Americas. This national program, sponsored by the Mexican government, focuses on achieving free and/or low-prevalence areas of four economical and quarantined Anastrepha species, i.e., A. ludens, A. obliqua, A. serpentina, and A. striata [14]. Therefore, three exotic braconid parasitoid species, namely D. longicaudata, D. tryoni [221], and F. arisanus [152] have been mass-reared at the Moscafrut facility in Metapa de Dominguez, Chiapas, Mexico and released. Of the three parasitoids species, 50 million pupae parasitized by $D$. longicaudata were produced weekly [222]. The first parasitoid augmentative releases in Mexico started in the late 80s, when an average of 1500 D. longicaudata parasitoids per ha. was released on almost 200 ha in the Valle of Mazapa de Madero, Chiapas, Mexico. Significant reductions in Anastrepha species were recorded with an average of $60 \%$ parasitism. This test was performed in a diverse range of Anastrepha species [223]. This was an important step towards implementing an IPM program in Anastrepha populations in Mexico. Consequently, new augmentative area-wide releases of the $D$. longicaudata, mass-reared, have been carried out by air or from the ground in different Mexican states $[14,15,222]$. Releases of $D$. longicaudata have been continuously focused on wild areas and backyard orchards to prevent fruit fly dispersion into commercial crops, which has caused substantial reductions in numbers of Anastrepha adults $[56,137,218,224,225]$. However, despite the good outcomes achieved with $D$. longicaudata, the Mexican National Program against Anastrepha spp. fruit flies has turned attention to the many neotropical-native parasitoid candidates for augmentative release with the chance of strategically increasing the mortality inflicted on pestiferous Anastrepha species. Thus, native parasitoid species better adapted to certain natural environmental conditions to low host densities, or that may attack other developmental stages of the pest, can be used to complement exotic parasitoid species [146,226-228]. Therefore, colonization of several neotropical parasitoid species and the mass-rearing of some of the species took place in Mexico $[18,57,229]$. Thus, the native C. haywardii is currently a suitable candidate for use with $D$. longicaudata in augmentative area-wide releases against Anastrepha spp. in Mexico [19,230,231].

The introduction of A. suspensa into Florida in 1965 led to the establishment of a biological control program which was developed by importing at least 11 parasitoid species from Hawaii, France, and Latin America between the early and late 1970s. Among introduced parasitoid species both $D$. longicaudata and the neotropical-native Doryctobracon areolatus (Szépligeti) has been successfully established into Florida [11]. Populations of $A$. suspensa decreased by $40 \%$ in some areas in the years following releases of the two braconid parasitoid species, but $A$. suspensa continued as a serious pest in Florida [159]. In view of this, a D. longicaudata mass-rearing and augmentative releases were carried out later, which generated significant reductions in A. suspensa populations in urban and suburban areas of Florida [144,232]. Because of these releases D longicaudata replaced D. areolatus as the major parasitoid of A. suspensa in the southern portion of Florida, while D. areolatus predominated in the northern sector [46,233].

In Brazil, the exotic D. longicaudata has shown the ability to adapt and settle in different environments, either in semi-arid or tropical areas, to control pestiferous Anastrepha species $[17,130$ $132,151]$. Augmentative releases of $D$. longicaudata against $A$. fraterculus in wild vegetation areas near citrus crops in the State of São Paulo, Brazil caused a reduction of 30\% in adult fly numbers [17]. Experimental studies under laboratory conditions showed the ability of $F$. arisanus to develop successfully in A. fraterculus eggs compared with C. capitata eggs [234]. This trait makes F. arisanus a complementary alternative to the use of $D$. longicaudata against $A$. fraterculus. 
Parasitoid species introduced into Argentina for the biological control of $C$. capitata were also released against $A$. fraterculus. Three exotic species, $D$. longicaudata, $A$. indica, and $P$. vindemmiae were established after releases in different Argentinean fruit-growing areas [16]. In addition to the introduced parasitoid species, both Brazil and Argentina followed the initiative of the Mexican National Fruit Fly Program regarding the use of neotropical parasitoids for Anastrepha control. Thus, several native parasitoid species were colonized and lab-reared to be evaluated as biocontrol agents of A. fraterculus in Brazil, i.e., Aganaspis pelleranoi $[235,236]$ and Doryctobracon brasiliensis $[237,238]$, and in Argentina, C. haywardii [20,97,239], A. pelleranoi [240,241], Doryctobracon crawfordi [240], and Opius bellus $[74,240]$.

In Peru, near to the border with Chile in the Department of Tacna, the National Medfly Program was releasing the parasitoid D. longicaudata weekly (reared in La Molina Facility in Lima) in the late 90 s in order to reduce C. capitata populations in olive orchards. More than $50 \%$ parasitism was achieved during the two years with the periodical massive releases of $D$. longicaudata. The market quality of olives was maintained at a high level with the implementation of an of IPM program against $C$. capitata in which the biological control played an important role.

There is only one document that records the introduction and releases of parasitoid species during the 1950s into California against $R$. completa, $R$. indifferens, and $R$. fausta [161], but the results of these releases were unsatisfactory [242]. Much later, evaluations of the parasitism capability of Psyttalia humilis (referred to as $P$. cf. concolor) on R. completa were carried out only under laboratoty conditions; this parasitoid was introduced into California for B. oleae biological control [202].

In the Western United States there are two parasitoids, Opius lectoides Gahan and O. downesi, which generally attack Rhagoletis zephyria Snow and R. pomonella in Oregon. About $60 \%$ of pupae were parasitized by these parasitoids on native host plants, while less than $2 \%$ on apple were attacked. Both species of Opius have short ovipositors, which may not be long enough to reach host larvae in the larger apple fruits. Alternatively, Diachasma allaeum has a much longer ovipositor and has been very successful in parasitizing larvae in apples in the Eastern United States [243].

One of the major concerns in the use of predators in pest control is intraguild predation [24]. Intraguild predation occurs among natural enemies in biological control systems, where one natural enemy (the intraguild predator) attacks another species of natural enemy (the intraguild prey), whereas they also compete for the same pest [27]. There are two types of intraguild predation (PGI) between predators and parasitoids: (1) The predator can directly predate the parasitoid, feeding from the immature phase then externally to the host and adult phase; (2) the predator can predate the parasitic host, directly consuming the host and, indirectly, the larva of the parasitoid [244]. The effect of the presence of intraguild predators on the intraguild prey was often negative, but sometimes no significant effect was detected [27]. Although predators are not the focus of fruit fly biological control programs, they have a very important role in conservation biological control, and it is necessary to intensify studies that evaluate or use agricultural techniques that do not affect an assemblage of predators of fruit flies, such as the use of selective pesticides.

Author Contributions: Conceptualization-F.R.M.G., J.C., O.E.L. and S.M.O.; writing - original draft preparation, F.R.M.G., J.C., O.E.L., S.M.O., and L.S; writing - review and editing, all authors; project administration, F.R.M.G.; funding acquisition, F.R.M.G. All authors have read and agreed to the published version of the manuscript.

Funding: This research received no external funding.

Acknowledgments: The authors would like to thank the the National Council of Technological and Scientific Development (CNPq) for the productivity scholarship provided to F.R.M.G.

Conflicts of Interest: The authors declare no conflict of interest. 
1. Norrbom, A.L.; Zucchi, R.A.; Hernandez-Ortiz, V. Phylogeny of the genera Anastrepha and Toxotrypana (Trypetinae: Toxotrypanini) based on morphology. In Fruit Flies (Tephritidae): Phylogeny and Evolution of Behavior; Aluja, M., Norrbom, A., Eds.; CRC Press: Boca Raton, FL, USA, 1999; pp. 299-342.

2. Rull, J. Distribución y Relevancia del género Rhagoletis en México. In Moscas de la Fruta: Fundamentos y Procedimientos para su Manejo; Montoya, P., Toledo, J., Hernández, E., Eds.; S y G Editores: México D.F., Mexico, 2020; pp. 165-184.

3. Liburd, O.E.; Stelinski, L.L.; Gut, L.J.; Thornton, G. Performance of various trap types for monitoring populations of cherry fruit fly (Diptera: Tephritidae) species. Environ. Entomol. 2001, 30, 82-88.

4. Glasgow, H. The host relations of our cherry fruit flies. J. Econ. Entomol. 1933, 26, 431-438.

5. Rice, R.; Phillips, P.; Stewart-Leslie, J.; Sibbett, G. Olive fruit fly populations measured in central and southern California. Calif. Agric. 2003, 57, 122-127.

6. Do, L.; Lemos, N.; Adaime, R.; De Jesus, C.R.; Da, E.; De Deus, G. New hosts of Bactrocera carambolae (diptera: Tephritidae) in Brazil. Fla. Entomol. 2014, 97, 841-843.

7. Hernández-Ortiz, V.; Guillén-Aguilar, J.; López, L. Taxonomía e Identificación de Moscas de la Fruta de Importancia económica en América. In Moscas de la Fruta: Fundamentos y Procedimientos para su Manejo; Montoya, P., Toledo, J., Hernández, E., Eds.; S y G Editores: México D.F., Mexico, 2010; pp. 49-80.

8. Vargas, R.I.; Leblanc, L.; Harris, E.J.; Mano, N.C. Regional suppression of Bactrocera fruit flies (Diptera: Tephritidae) in the pacific through biological control and prospects for future introductions into other areas of the world. Insects 2012, 3, 727-742.

9. Sivinski, J. The past and potential of biological control of fruit flies. In Fruit Fly Pest. A World Assessment of their Biology and Management; McPheron, B.A., Steck, G.J., Eds.; St. Lucie Press: Delray Beach, FL, USA, 1996; pp. 369-375.

10. Purcell, M.F.; Herr, J.C.; Messing, R.H.; Wong, T.T.Y. Interactions between augmentatively released Diachasmimorpha longicaudata and a complex of opiine parasitoids in a commercial guava orchard. Biocontrol Sci. Technol. 1998, 8, 139-151.

11. Ovruski, S.; Aluja, M.; Sivinski, J.; Wharton, R. Hymenopteran Parasitoids on Fruit Infesting Tephritidae (Diptera) in Latin America and the Southern United States: Diversity, Distribution, Taxonomic Status and their use in Fruit Fly Biological Control. Integr. Pest Manag. Rev. 2000, 5, 81-107.

12. Stelinski, L.L.; Pelz, K.S.; Liburd, O.E. Field observations quantifying attraction of the parasitic wasp, Diachasma alloeum (Hymenoptera: Braconidae) to blueberry fruit infested by the blueberry maggot fly, Rhagoletis mendax (Diptera: Tephritidae). Fla. Entomol. 2004, 87, 124-129.

13. Dias, N.P.; Zotti, M.J.; Montoya, P.; Carvalho, I.R.; Nava, D.E. Fruit fly management research: A systematic review of monitoring and control tactics in the world. J. Crop Prot. 2018, 112, 187-200.

14. Montoya, P.; Toledo, J. Estrategias de control biológico. In Moscas de la Fruta: Fundamentos y Procedimientos para su Manejo; Montoya, P., Toledo, J., Hernández, E., Eds.; S y G Editores: México, D.F., Mexico, 2020; pp. 259-276.

15. Montoya, P.; Cancino, J.; Zenil, M.; Santiago, G.; Gutierrez, J.M. The augmentative biological control component in the Mexican National Campaign against Anastrepha spp. Fruit Flies. In Area-Wide Control of Insect Pests. Research to Field Implementation; Vreysen, M.J.B., Robinson, A.S., Hendrichs, J., Eds.; Springer: Dordrecht, The Netherlands, 2007; pp. 661-670.

16. Ovruski, S.M.; Schliserman, P. Biological control of tephritid fruit flies in Argentina: Historical review, current status, and future trends for developing a parasitoid mass-release program. Insects 2012, 3, 870888.

17. Garcia, F.R.M.; Ricalde, M.P. Augmentative biological control using parasitoids for fruit fly management in Brazil. Insects 2013, 4, 55-77.

18. Aluja, M.; Sivinski, J.; Ovruski, S.; Guillén, L.; López, M., Cancino, J.; Ruíz, L. Colonization and domestication of seven species of native New World hymenopterous larval-prepupal and pupal fruit fly (Diptera: Tephritidae) parasitoids. Biocontrol Sci. Technol. 2009, 19, 49-79.

19. Cancino, J.; Montoya, P.; Barrera, J.F.; Aluja, M.; Liedo, P. Parasitism by Coptera haywardi and Diachasmimorpha longicaudata on Anastrepha flies with different fruits under laboratory and field cage conditions. BioControl 2014, 59, 287-295.

20. Núñez-Campero, S.R.; Suárez, L.; Buonocore Biancheri, M.J.; Cancino, J.; Murúa, F.; Molina, D.; Laria, O.; Aluja, M.; Ovruski, S.M. Host suitability and fitness-related parameters in Coptera haywardi (Hymenoptera: 
Diapriidae) reared on irradiated Ceratitis capitata (Diptera: Tephritidae) pupae stemming from the tsl Vienna-8 genetic sexing strain. J. Econ. Entomol. 2020, doi:10.1093/jee/toaa109.

21. Bokonon-Ganta, A.H.; Wang, X.G.; Messing, R.H. Biological control of tephritid fruit flies in Hawaii with special reference to the newly discovered egg-larval parasitoid, Fopius ceratitivorus (Wharton). Proc. Hawaii. Entomol. Soc. 2007, 39, 87-94.

22. Aluja, M.; Sivinski, J.; Rull, J.; Hodgson, P.J. Behavior and predation of fruit fly larvae (Anastrepha spp.) (Diptera: Tephritidae) after exiting fruit in four types of habitats in Tropical Veracruz, Mexico. Environ. Entomol. 2005, 34, 1507-1516.

23. Fernandes, W.D.; Sant'Ana, M.V.; Raizer, J.; Lange, D. Depredación de larvas de mosca de la fruta Anastrepha (Diptera: Tephritidae) por hormigas en el bosque. Psique 2012, 10, 83-89.

24. Garcia, F.R.M.; Brida, A.L.; Martins, L.N.; Abeijon, L.M.; Lutinski, C.J. Biological Control of Fruit Flies of the genus Anastrepha (Diptera: Tephritidae): Current Status and Perspectives. In Biological Control: Methods, Applications and Challenges, 1st ed.; Nova Science Publishers: Hauppauge, NY, USA, 2017; pp. $29-71$.

25. Abeijon, L.M.; Kruger, A.P.; Lutinski, J.; Garcia, F.R.M. Can ants contribute to the conservative biological control of the South American fruit fly? Biosci. J. 2019, 35, 941-948.

26. Hodgson, P.J.; Sivinski, J.; Quintero, G.; Aluja, M. Depth of pupation and survival of fruit fly (Anastrepha spp.: Tephritidae) pupae in a range of agricultural habitats. Environ. Entomol. 1998, 27, 1310-1314.

27. Janssen, A.; Montserrat, M.; Hillerislambers, R.; Roos, A.M.; Pallini, A.; Sabelis, M.W. Intraguild predation usually does not disrupt biological control. In Trophic and Guild Interactions in Biological Control, 1st ed.; Brodeur, J., Boivin, G., Eds.; Springer: Dordrecht, The Netherlands, 2006; pp. 21-44.

28. Brodeur, J.; Boivin, G. Functional ecology of immature parasitoids. Annu. Rev. Entomol. 2004, 49, $27-49$.

29. Alvarado, L.; Medianero, E. Especies de parasitoides asociados a moscas de la fruta del género Anastrepha (Diptera: Tephritidae) en Panamá, república de Panamá. Scientia 2015, 25, 47-62.

30. Canal, N.A.; Zucchi, R.A.; da Silva, N.M.; Leonel, F.L., Jr. Reconocimiento de las especies de parasitoides (Hymenoptera: Braconidae) de moscas de las frutas (Diptera: Tephritidae) en dos municipios del Estado de Amazonas, Brasil. Boletín Mus. Entomol. Univ. Val. 1994, 2, 1-17.

31. Jesus-Barros, C.R.; Adaime, R.; Oliveira, M.N.; Silva, W.R.; Costa Neto, S.V.; Souza Filho, M.F. Anastrepha (Diptera: Tephritidae) species, their hosts and parasitoids (Hymenoptera: Braconidae) in five municipalities of the state of Amapá, Brazil. Fla. Entomol. 2012, 95, 694-705.

32. Leonel, F.L., Jr.; Zucchi, R.A.; Wharton, R.A. Distribution and tephritid hosts (Diptera) of braconid parasitoids (Hymenoptera) in Brazil. Int. J. Pest Manag. 1995, 41, 208-213.

33. Marinho, C.F.; Costa, V.A.; Zucchi, R.A. Annotated checklist and illustrated key to braconid parasitoids (Hymenoptera, Braconidae) of economically important fruit flies (Diptera, Tephritidae) in Brazil. Zootaxa 2018, 4527, 21-36.

34. Schliserman, P.; Ovruski, S.; DeColl, O.R.; Wharton, R. Diversity and abundance of hymenopterous parasitoids associated with Anastrepha fraterculus (Diptera: Tephritidae) in native and exotic host plants in Misiones, Northeastern Argentina. Fla. Entomol. 2010, 93, 175-182.

35. Silva, R.A.; Lima, A.L.; Xavier, S.L.O.; Silva, W.R.; Marinho, C.F.; Zucchi, R.A. Anastrepha species (Diptera: Tephritidae), their hosts and parasitoids in southern Amapá State, Brazil. Biota Neotrop. 2011, 11, 431-436.

36. Zucchi, R.A.; Moraes, R.C.B. Base de dados dos hospedeiros e parasitoides de Ceratitis capitata ((Wied.) (Diptera, Tephritidae) no Brasil. In Proceedings of the XXIV Congresso Brasileiro de Entomologia, Curitiba, Brazil, 16-22 September 2012.

37. Forbes, A.A.; Hood, G.R.; Feder, J.L. Geographic and ecological overlap of parasitoid wasps associated with the Rhagoletis pomonella (Diptera: Tephritidae) species complex. Ann. Entomol. Soc. Am. 2010, 103, 908-915.

38. Greathead, D.J.; Greathead, A.H. Biological control of insect pests by insect parasitoids and predators: The BIOCAT database. Biocontrol 1992, 13, 61-68.

39. Wharton, R.; Ward, L.; Miko, I. New Neotropical species of Opiinae (Hymenoptera, Braconidae) reared from fruit-infesting and leaf-mining Tephritidae (Diptera) with comments on the Diachasmimorpha Mexicana species group and the genera Lorenzopiusand tubiformopius. ZooKeys 2012, 243, $27-82$.

40. Feder, J.L. The effects of parasitoids on sympatric host races of Rhagoletis pomonella (Diptera: Tephritidae). Ecology 1995, 76, 801-813.

41. Hagen, K.S.; Tassan, R.L.; Fong, M.; Aliniazee, M.T. Walnut husk fly, Rhagoletis complete. In Biological Control in the Western United States. Accomplishments and Benefits of Regional Research Project W-84, 
1964-1989; Nechols, J.R., Ed.; University of California, Division of Agriculture and Natural Resources, Publication: Parkville, CA, USA, 1995; pp. 224-227.

42. Marinho, C.F.; Cônsoli, F.L.; Penteado-Dias, A.M.; Zucchi, R.A. Description of two new species closely related to Doryctobracon areolatus (Szépligeti, 1911) (Hymenoptera, Braconidae), based on morphometric and molecular analyses. Zootaxa 2017, 4353, 467-484.

43. Alvarenga, C.D.; Matrangolo, C.A.R.; Lopes, G.N.; Silva, M.A.; Lopes, E.N.; Alves, D.A.; Nascimento, A.S.; Zucchi, R.A. Moscas-das-frutas (Diptera: Tephritidae) e seus parasitóides em plantas hospedeiras de três municípios do norte do estado de Minas Gerais. Arq. Inst. Biol. 2009, 76, 195-204.

44. Canal, N.A.; Zucchi, R.A. Parasitóides-Braconidae. In Moscas-das-frutas de Importância Econômica no Brasil. Conhecimento Básico e Aplicado; Malavasi, A., Zucchi, R.A., Eds.; Holos-FAPESP: São Paulo, Brazil, 2000; pp. 119-126.

45. Carrejo, N.S.; Gonzalez, O.R. Parasitoids reared from species of Anastrepha (Diptera: Tephritidae) in Valle del Cauca, Colombia. Fla. Entomol. 1999, 82, 113-118.

46. Eitam, A.; Sivinski, J.; Holler, T.; Aluja, M. Biogeography of braconid Parasitoids of the Caribbean fruit fly (Diptera: Tephritidae) in Florida. Ann. Entomol. Soc. Am. 2004, 97, 928-939.

47. Eskafi, F.M. Parasitism of fruit flies Ceratitis capitata and Anastrepha spp. (Diptera: Tephritidae) in Guatemala. Entomophaga 1990, 35, 355-362.

48. Garcia, F.R.M.; Corseuil, E. Native hymenopteran parasitoids associated with fruit flies (Diptera: Tephritidae) in Santa Catarina State, Brazil. Fla. Entomol. 2004, 87, 517-521.

49. Jiron, L.F.; Mexon, R.G. Parasitoid hymenopterans of Costa Rica: Geographical distribution of species associated with fruit flies (Diptera: Tephritidae). Entomophaga 1989, 34, 53-60.

50. Katiyar, K.P.; Camacho, J.; Geraud, F.; Matheus, R. Parasitoides hymenópteros de moscas de las frutas (Diptera: Tephritidae) en la región occidental de Venezuela. Rev. Fac. Agron. 1995, 12, 303-312.

51. Leonel, F.L., Jr.; Zucchi, R.A.; Canal Daza, N.A. Parasitismo de moscas-das-frutas (Diptera: Tephritidae) por Braconidae (Hymenoptera) em duas localidades do Estado de São Paulo. An. Soc. Entomol. Bras. 1996, 25, 199-206.

52. López, M.; Aluja, M.; Sivinski, J. Hymenopterous larval-pupal and pupal parasitoids of Anastrepha flies (Diptera: Tephritidae) in Mexico. Biol. Control 1999, 15, 119-129.

53. Marsaro, A.L., Jr.; Silva, R.A.; Silva, W.R.; Lima, C.R.; Flores, A.S.; Ronchi-Teles, B. New records of Anastrepha (Diptera: Tephritidae), its hosts and parasitoids in the Serra do Tepequém, Roraima state, Brazil. Rev. Agric. 2010, 85, 15-19.

54. Marsaro, A.L., Jr.; Adaime, R.; Ronchi-Teles, B.; Lima, C.R.; Pereira, P.R.V.S. Anastrepha species (Diptera: Tephritidae), their hosts and parasitoids in the extreme north of Brazil. Biota Neotrop. 2011, 11, 117-124.

55. Medianero, E.; Korytkowski, C.H.; Campo, C.; De León, C. Hymenoptera parasitoides asociados a Anastrepha (Diptera: Tephritidae) en Cerro Jefe y Altos de Pacora, Panamá. Rev. Colomb. Entomol. 2006, 32, 136-139.

56. Montoya, P.; López, P.; Cruz, J.; López, F.; Cadena, C.; Cancino, J.; Liedo, P. Effect of Diachasmimorpha longicaudata releases on the native parasitoid guild attacking Anastrepha spp. larvae in disturbed zones of Chiapas, Mexico. BioControl 2017, 62, 581-593.

57. Murillo, F.D.; Cabrera-Mireles, H.; Barrera, J.F.; Liedo, P.; Montoya, P. Doryctobracon areolatus (Hymenoptera, Braconidae) a parasitoid of early developmental stages of Anastrepha obliqua (Diptera, Tephritidae). J. Hymenopt. Res. 2015, 46, 91-105.

58. Ovruski, S.M.; Fuentes, S.; Núñez, F.; Granados Zúñiga, J.G. Himenópteros parasitoides de moscas de la frura (Diptera: Tephritidae) presentes en la República de El Salvador. Rev. Ing. Agron. 1996, 14, 8-14.

59. Ovruski, S.M.; Schliserman, P.; Núñez-Campero, S.R.; Oroño, L.; Bezdjian, L.; Albornoz Medina, P.; Van Nieuwenhove, G.A. Survey of hymenopterous larval-pupal parasitoids associated with Anastrepha fraterculus and Ceratitis capitata (Diptera: Tephritidae) infesting wild guava (Psidium guajava) and peach (Prunus persica) in the southernmost section of the bolivian Yungas forest. Fla. Entomol. 2009, 92, 269-275.

60. Ovruski, S.M.; Schliserman, P.; Aluja, M. Occurrence of diapause in neotropical parasitoids attacking Anastrepha fraterculus (Diptera: Tephritidae) in a subtropical rainforest from Argentina. Austral Entomol. 2016, 55, 2-23.

61. Schliserman, P.; Aluja, M.; Rull, J.; Ovruski, S.M. Temporal diversity and abundance patterns of parasitoids of fruit-infesting Tephritidae (Diptera) in the Argentinean Yungas: Implications for biological control. Environ. Entomol. 2016, 45, 1184-1198. 
62. Silva, J.G.; Dutra, V.S.; Santos, M.S.; Silva, N.O.; Vidal, D.B.; Nink, R.A.; Guimarães, J.A.; Araujo, E.L. Diversity of Anastrepha spp. (Diptera: Tephritidae) and associated braconid parasitoids from native and exotic hosts in southeastern Bahia, Brazil. Environ. Entomol. 2010, 39, 1457-1465.

63. Ovruski, S.M.; Schliserman, P.; Aluja, M. Indigenous parasitoids (Hymenoptera) attacking Anastrepha fraterculus and Ceratitis capitata (Diptera: Tephritidae) in native and exotic host plants in Northwestern Argentina. Biol. Control 2004, 29, 43-57.

64. Ovruski, S.M.; Wharton, R.A.; Schliserman, P.; Aluja, M. Abundance of Anastrepha fraterculus (Diptera: Tephritidae) and its associated native parasitoids (Hymenoptera) in "feral" guavas growing in the endangered northernmost Yungas forest of Argentina with an update on the taxonomic status of opiine parasitoids previously reported in this country. Environ. Entomol. 2005, 34, 807-818.

65. Pirovani, V.D.; Martins, D.S.; Souza, S.A.S.; Uramoto, K.; Ferreira, P.S.F. Moscas-das-frutas (Diptera: Tephritidae), seus parasitoides e hospedeiros em Viçosa, zona da mata mineira. Arq. Inst. Biol. São Paulo 2010, 77, 727-733.

66. Núñez Bueno, L.; Gómez Santos, R.; Guarín, G.; León, G. Moscas de las frutas (Díptera: Tephritidae) y parasitoides asociados con Psidium guajava L. y Coffea arabica L. en tres municipios de la Provincia de Vélez (Santander, Colombia). Parte 2: Identificación y evaluación de parasitoides del Orden Hymenoptera. Rev. Corpoica 2004, 5, 13-21.

67. González-Hernández, A.; Tejada, L.O. Fluctuación de la población de Anastrepha ludens (Loew) y de sus enemigos naturales en Sargentia greggi S. Watts. Folía Entomol. Mex. 1979, 41, 49-60.

68. Ruiz, L.; Oropeza, A.; Toledo, J. Nuevas asociaciones de especies de parasitoides y Anastrepha distincta (Diptera: Tephritidae) en el Soconusco, Chiapas, México. Acta Zool. Mex. 2011, 27, 813-818.

69. Stibick, J.N.L. Natural Enemies of True Fruit Flies (Tephritidae); United States Department of Agriculture, Animal and Plant Health Inspection Service: Riverdale, MD, USA, 2004; 86p.

70. Wharton, R.A.; Marsh, P.M. New world Opiinae (Hymenoptera: Braconidae) parasitic on Tephritidae (Diptera). J. Wash. Acad. Sci. 1978, 68, 147-167.

71. Dutra, V.S.; Ronchi-Teles, B.; Garcia, M.V.B.; Adaime, R.; Silva, J.S. Native hosts and parasitoids associated with Anastrepha fractura and other Anastrepha species (Diptera: Tephritidae) in the Brazilian Amazon. Fla. Entomol. 2013, 96, 270-273.

72. Guagliumi, P. La mosca del mediterráneo (Ceratitis capitata Wied. Dip.: Trypetidae) in Venezuela. Rev. Agron. Subtrop. 1963, 57, 364-369.

73. Hernández-Ortiz, V.; González, H.D.; Escalante-Tio, A.; Manrique-Saide, P. Hymenopteran parasitoids of Anastrepha fruit flies (Diptera: Tephritidae) reared from different hosts in Yucatan, Mexico. Fla. Entomol. 2006, 89, 508-514.

74. Schliserman, P.; Van Nieuwenhove, G.A.; Bezdjian, L.P.; Albornoz Medina, P.; Escobar, L.I.; Buonocore Biancheri, M.J.; Altamirano, J.; Aluja, M.; Ovruski, S.M. Bionomics of Opius bellus (Hymenoptera: Braconidae), an endoparasitoid of Anastrepha fraterculus (Diptera: Tephritidae) in fruitgrowing areas of Northwestern Argentina. Biocontrol Sci. Technol. 2014, 24, 375-388.

75. Wharton, R.A.; Gilstrap, F.E.; Rhodei, R.H.; Fischel-M., M.; Hart, W.G. Hymenopterous egg-pupal and larval-pupal parasitoids of Ceratitis capitata and Anastrepha spp. (Diptera: Tephritidae) in Costa Rica. Entomophaga 1981, 26, 285-290.

76. García-Medel, D.; Sivinski, J.; Diáz-Fleischer, F.; Ramirez-Romero, R.; Aluja, M. Foraging behavior by six fruitfly parasitoids (Hymenoptera: Braconidae) released as single- or multiple-species cohorts infield cages: Influence of fruit location and host density. Biol. Control 2007, 43, 12-22.

77. Hernández-Ortíz, V.; Pérez-Alonso, R.; Wharton, R.A. Native parasitoids associated with the genus Anastrepha (Dipt.: Tephritidae) in Los Tuxtlas, Veracruz, Mexico. Entomophaga 1994, 39, 171-178.

78. Sivinski, J.; Jerónimo, F.; Holler, T. Development of aerial releases of Diachasmimorpha tryoni (Cameron) (Hymenoptera: Braconidae), a parasitoid that attacks the Mediterranean fruitfly, Ceratitis capitata (Weidemann) (Diptera: Tephritidae), in the Guatemalan highlands. Biocontrol Sci. Technol. 2000, 10, 15-25.

79. Wharton, R.A. Variation in Opius hirtus Fischer and discussion of Desmiostoma Foerster (Hymenoptera: Braconidae). Proc. Entomol. Soc. Wash. 1983, 85, 327-330.

80. Araujo, E.L.; Fernandes, E.C.; Silva, R.I.R.; Ferreira, A.D.C.L.; Costa, V.A. Parasitoides (Hymenoptera) de moscas-das-frutas (Diptera: Tephritidae) no semiárido do estado do Ceará, Brasil. Rev. Bras. Frutic. 2015, 37, 610-616. 
81. Bartlett, K.A. The introduction and colonization in Puerto Rico of benefical insects parasitic on West Indian fruit flies. J. Agric. Univ. Puerto Rico 1941, 25, 25-31.

82. Borges-Soto, M.; Rubial, M.R.; Terrero, E.R.E.; Saber-Muñoz, B. Implementation of an Anastrepha spp. Riskprotocol for mango export industry in Cuba. In Area-Wide Management of Fruit Fly Pests; Pérez-Staples, D., Diaz-Fleischer, F., Montoya, P., Vera, M.T., Eds.; CRC Press: Boca Raton, FL, USA, 2020; pp. 333-342.

83. Aguiar-Menezes, E.L.; Menezes, E.B. Natural occurence of parasitoids of Anastrepha spp. Schiner, 1868 (Diptera: Tephritidae) in different host plants, in Itaguai (RJ), Brazil. Biol. Control 1997, 8, 1-6.

84. Nasca, A.J. Parásitos de moscas de los frutos establecidos en algunas zonas de Tucumán. Rev. Agric. N. O. Arg. 1973, 10, 31-43.

85. Nicácio, J.N.; Uchôa, M.A.; Faccenda, O.; Guimarães, J.A.; Marinho, C.F. Native larval parasitoids (Hymenoptera) of frugivorous Tephritoidea (Diptera) in South Pantanal region, Brazil. Fla. Entomol. 2011, 94, 407-419.

86. Ovruski, S.M.; Schliserman, P.; DeColl, O.R.; Peñaloza, C.; Oroño, L.; Colin, C. The establishment of Aceratoneuromyia indica (Hymenoptera: Eulophidae) in three biogeographical regions of Argentina. Fla. Entomol. 2006, 89, 270-273.

87. Sivinski, J.; Aluja, M.; López, M. The spatial and temporal distributions of parasitoids of Mexican Anastrepha species (Diptera: Tephritidae) within canopies of fruit trees. Ann. Entomol. Soc. Am. 1997, 90, 604-618.

88. Muñiz-Reyes, E.; Lomelí-Flores, J.R.; Sánchez-Escudero, J. Parasitoides nativos de Rhagoletis pomonella Walsh (Diptera: Tephritidae) en tejocote Crataegus spp. en el centro de México. Acta Zool. Mex. 2011, 27, 425-440.

89. Rull, J.; Wharton, R.; Feder, J.L.; Guillen, L.; Sivinski, J.; Forbes, A.; Aluja, M. Latitudinal variation in parasitoid guild composition and parasitism rates of North American hawthorn infesting Rhagoletis. Environ. Entomol. 2009, 38, 588-599.

90. Canal, N.A.; Zucchi, R.A.; Nora, I. Considerações sobre Opius tomoplagiae Lima, 1938 (Hym., Braconidae). In Proceedings of the 15 Congresso Brasileiro de Entomologia, Resumos, Caxambu, MG, Brazil, 12-17 March 1995; p. 775.

91. Nora, I.; Zucchi, R.A.; Branco, E.S. Levantamento de parasitóides (Hym., Braconidae) de Anastrepha (Dip., Tephritidae) na Região do Alto Vale do Rio do Peixe, SC. In Proceedings of the 15 Congresso Brasileiro de Entomologia, Resumos, Caxambu, MG, Brazil, 12-17 March 1995; pp. 298.

92. Nora, I.; Hickel, E.R.; Prando, H.F. Moscas-das-frutas nos estados brasileiros: Santa Catarina. In MoscasDas-Frutas de Importância Econômica no Brasil: Conhecimento Básico e Aplicado; Malavasi, A., Zucchi, R.A., Eds.; Holos Editora: Ribeirão Preto, Brasil, 2000; pp. 271-275.

93. Aguiar-Menezes, E.L.; Menezes, E.B.; Loiácono, M.S. First record of Coptera haywardi Loiácono (Hymenoptera: Diapriidae) as a parasite of fruit-infesting Tephritidae (Diptera) in Brazil. Neotrop. Entomol. 2003, 32, 355-358.

94. García, J.L.; Montilla, R. Coptera haywardi Loiácono (Hymenoptera: Diapriidae) parasitoide de pupas de Anastrepha spp. (Diptera: Tephritidae) en Venezuela. Entomotropica 2001, 16, 191-195.

95. Guillén, L.; Aluja, M.; Equihua, M.; Sivinski, J. Performance of two fruit fly (Diptera: Tephritidae) pupal parasitoids (Coptera haywardi [Hymenoptera: Diapriidae] and Pachycrepoideus vindemiae [Hymenoptera: Pteromalidae]) under different environmental soil conditions. Biol. Control 2002, 23, 219-227.

96. Loiácono, M.S. Notas sobre Diapriinae neotropicales (Hymenoptera: Diapriidae). Rev. Soc. Entomol. Argen. 1981, 40, 237-241.

97. Núñez-Campero, S.R.; Ovruski, S.M.; Aluja, M. Survival analysis and demographic parameters of the pupal parasitoid Coptera haywardi (Hymenoptera: Diapriidae), reared on Anastrepha fraterculus (Diptera: Tephritidae). Biol. Control 2012, 61, 40-46.

98. Silva, B.K.A.; Silva, H.M.; Fernandes, E.C.; Costa, V.A.; Araujo, E.L. Pupal parasitoids associated with Ceratitis capitata (Wiedemann) (Diptera: Tephritidae) in a semiarid environment in Brazil. Rev. Bras. Entomol. 2020, 64, 1-6.

99. Cruz, P.P.; Neutzling, A.S.; Garcia, F.R.M. Primeiro registro de Trichopria anastrephae, parasitoide de moscas-das-frutas, no Rio Grande do Sul. Ciência Rural 2011, 41, 1297-1299.

100. Turica, A.; Mallo, R.G. Observaciones sobre la población de las “Tephritidae” y sus endoparásitos en algunas regiones citrícolas argentinas. IDIA 1961, 6, 145-161. 
101. Mena-Correa, J.; Sivinski, J.; Anzures-Dadda, A.; Ramírez-Romero, R.; Gates, M.; Aluja, M. Consideration of Eurytoma sivinskii Gates and Grissell, a eurytomid (Hymenoptera) with unusual foraging behaviors, as a biological control agent of tephritid (Diptera) fruit flies. Biol. Control 2010, 53, 9-17.

102. Ovruski, S.M.; Wharton, R.A.; Rull, J.; Guillen, L. Aganaspis alujai (Hymenoptera: Figitidae: Eucoilinae), a new species attacking Rhagoletis (Diptera: Tephritidae) in the neotropical region. Fla. Entomol. 2007, 90, 626634.

103. Rull, J.; Lasa, R.; Guillén, L.; Aluja, M. Effect of winter length on duration of dormancy and survival of Rhagoletis completa (Diptera: Tephritidae) and associated parasitoids from Northeastern Mexico. J. Insect Sci. 2019, 19, 7.

104. Guimarães, J.A.; Diaz, N.B.; Zucchi, R.A. Parasitoides-Figitidae (Eucoilinae). In Moscas-Das-Frutas de Importância Econômica no Brasil. Conhecimento básico e Aplicado; Malavasi, A., Zucchi, R.A., Eds.; HolosFAPESP: Ribeirão Preto, Brazil, 2000; pp. 127-134.

105. Wharton, R.A.; Ovruski, S.M.; Gilstrap, F.E. Neotropical Eucoilidae (Cynipoidea) associated with fruit infesting Tephritidae, with new records from Argentina, Bolivia and Costa Rica. J. Hym. Res. 1998, 7, 102115.

106. Aluja, M.; Guillén, J.; Liedo, P.; Cabrera, M.; Ríos, E.; de la Rosa, G.; Celedonio, H. Fruit infesting tephritids (Dipt.: Tephritidae) and associated parasitoids in Chiapas, Mexico. Entomophaga 1990, 35, 39-48.

107. Guimarães, J.A. Espécies de Eucoilinae (Hymenoptera: Figitidae) Parasitóides de Larvas Frugívoras (Diptera: Tephritidae e Lonchaeidae) no Brasil; Dissertação de Mestrado, ESALQ/USP: Piracicaba, Brazil, 1998.

108. Aluja, M., Ovruski, S.M., Guillén, L., Oroño, L.E.; Sivinski, J. Comparison of the host searching and oviposition behaviors of the tephritid (Diptera) parasitoids Aganaspis pelleranoi and Odontosema anastrephae (Hymenoptera: Figitidae, Eucoilinae). J. Insect Behav. 2009, 22, 423-451.

109. López, P.; Cancino, J.; Montoya, P. Natural parasitism and parasitoid releases to control Anastrepha obliqua (Diptera: Tephritidae) infesting Spondias spp. (Anacardaceae) in Chiapas, México. In Area-Wide Management of Fruit Flies; Pérez-Staples, D., Díaz-Fleisher, F., Montoya, P., Vera, M.T., Eds.; CRC Press: Boca Raton, FL, USA, 2020; pp. 268-275.

110. Fernandes, D.R.R.; Vacari, A.M.; Araujo, E.L.; Guimarães, J.A.; De Bortoli, S.A.; Perioto, N.W. Frugivorous flies (Diptera: Tephritidae and Lonchaeidae) and native parasitoids (Hymenoptera) associated with Pouteria caimito (Sapotaceae) in Brazil. Fla. Entomol. 2013, 96, 255-257.

111. Souza, A.R.; Lopes-Mielezrski, G.N.; Lopes, E.N.; Querino, R.B.; Corsato, C.D.A.; Giustolin, T.A.; Zucchi, R.A. Hymenopteran Parasitoids Associated with Frugivorous Larvae in a Brazilian Caatinga-Cerrado Ecotone. Environ. Entomol. 2012, 41, 233-237.

112. Hoffmeister, T.S. Rhagoletis pomonella (Walsh), apple maggot (Diptera: Tephritidae). In Biological Control Programmes in Canada, 1981-2000; Mason, P.G., Huber, J.T., Eds.; CABI Publishing: Wallingford, UK, 2002; pp. 238-241.

113. Baranowski, R.; Swanson, R.W. Introduction of Parachasma (opius) cereus (Hymenoptera: Braconidae) into Florida as a parasite of Anastrepha suspensa (Diptera: Tephritidae). Fla. Entomol. 1970, 53, 161-162.

114. Uchôa, M.A. Fruit Flies (Diptera: Tephritoidea): Biology, host plants, natural enemies, and the implications to their natural control. In Integrated Pest Management and Pest Control. Current and Future Tactics; Larramendy, M.L.; Soloneski, S.; Eds.; Intech Open: London, UK, 2012; pp. 271-300.

115. Haramoto, F.H.; Bess, H.A. Recent studies on the abundance of the Oriental and Mediterranean fruit flies and the status of their parasites. Proc. Hawaii. Entomol. Soc. 1970, 20, 551-566.

116. Bautista, R.C.; Harris, E.J.; Lawrence, P.O. Biology and rearing of the fruit fly parasitoid Biosteres arisanus: Clues to insectary propagation. Entomol. Exp. Appl. 1998, 89, 79-85.

117. Sime, K.R.; Daane, K.M.; Andrews, J.W.; Hoelmer, K.A.; Pickett, C.H.; Nadel, H.; Johnson, M.W.; Messing, R.H. The biology of Bracon celer as a parasitoid of the olive fruit fly. BioControl 2006, 51, 553-567.

118. Nadel, H.; Daane, K.M.; Hoelmer, K.A.; Pickett, C.H.; Johnson, M.W. Non-target host risk assessment of the idiobiont parasitoid, Bracon celer (Hymenoptera: Braconidae), for biological control of olive fruit fly in California. Biocontrol Sci. Technol. 2009, 19, 701-715.

119. Steck, G.J.; Gilstrap, F.E.; Wharton, R.A.; Hart, W.G. Braconid parasitoids of Tephritidae (Diptera) infesting coffee and other fruits in West-Central Africa. Entomophaga 1986, 31, 59-67.

120. Clausen, C.P. Introduced Parasites and Predators of Arthropod Pests and Weeds: A World Review; Agriculture Handbook No. 480; USDA: Washington, DC, USA, 1978; 551p. 
121. Rendón, P.; Sivinski, J.; Holler, T.; Bloem, K.; López, M.; Marínez, M.; Aluja, M. The effects of sterile males and two braconid parasitoids, Fopius arisanus (Sonan) and Diachasmimorpha krausii (Fullaway) (Hymenoptera), on caged populations of Mediterranean fruit flies, Ceratitis capitata (Wied.) (Diptera: Tephritidae) at various sites in Guatemala. Biol. Control 2006, 36, 224-231.

122. Sime, K.R.; Daane, K.M.; Nadel, H.; Funk, C.S.; Messing, R.H.; Andrews, J.W.; Johnson, M.W.; Pickett, C.H. Diachasmimorpha longicaudata and D. kraussii (Hymenoptera: Braconidae), potential parasitoids of the olive fruit fly. Biocontrol Sci. Technol. 2006, 16, 169-179.

123. Bokonon-Ganta, A.H.; McQuate, G.T.; Messing, R.H.; Jang, E.B. Release and establishment of the parasitoid Diachasmimorpha kraussii against the tephritid fruit fly Bactrocera latifrons in Hawaii. J. Insect Sci. 2013, $13,7$. doi:10.1673/031.013.0701

124. Schliserman, P.; Ovruski, S.M.; DeColl, O.R. The recovery and permanent establishment of Diachasmimorpha longicaudata (Hymenoptera: Braconidae) in Misiones, northeastern Argentina. Fla. Entomol. 2003, 86, 491-492.

125. Ovruski, S.M.; Bezdjian, L.P.; van Nieuwenhove, G.A.; Albornoz-Medina, P.; Schliserman, P. Host preference by Diachasmimorpha longicaudata (Hymneoptera: Braconidae) reared on larvae of Anastrepha fraterculus and Ceratitis capitata (Diptera: Tephritidae). Fla. Entomol. 2011, 94, 195-200.

126. Sánchez, G.; Murúa, F.; Suárez, L.; van Nieuwenhove, G.; Taret, G.; Pantano, V.; Ovruski, S.M. Augmentative releases of Diachasmimorpha longicaudata (Hymenoptera: Braconidae) for Ceratitis capitata (Diptera: Tephritidae) control in a fruit-growing region of Argentina. Biol. Control 2016, 103, 101-107.

127. Suárez, L.; Buonocore Biancheri, M.J.; Murúa, F.; Bilbao, M.; García, M.; Cancino, J.; Martin, O.; Molina, D.; Laría, O.; Ovruski, S.M. Effects of host age and radiation dose in Diachasmimorpha longicaudata (Hymenoptera: Braconidae) mass reared on Medfly larvae of the tsl Vienna 8 genetic sexing strain. Biol. Control 2019, 130, 51-59.

128. Suárez, L.; Buonocore Biancheri, M.J.; Sánchez, G.; Murúa, F.; Funes, C.F.; Kirschbaum, D.S.; Molina, D.; Laría, O.; Ovruski, S.M. Effects of releasing two Diachasmimorpha longicaudata population lines for the control of Ceratitis capitata infesting three key host fruit species. Biol. Control 2019, 133, 58-65.

129. Pruett, C.J.H. Biological control in Bolivia: History and development. In El control biológico en América Latina; Zapater, M.C., Ed.; SRNT, IOBC: Buenos Aires, Argentina, 1996; pp. 17-24.

130. Walder, J.M.M. Produção de moscas-das-frutas e seus inimigos naturais: Associação de moscas estéreis e controle biológico. In Controle biológico no Brasil: Parasitóides e Predadores; Parra, J.R.P., Botelho, P.S.M., Corrêa-Ferreira, B.S., Bento, J.M.S., Eds.; Manole: São Paulo, Brazil, 2002; pp.181-190.

131. Carvalho, R.S. Avaliação das liberações inoculativas do parasitóide exótico Diachasmimorpha longicaudata (Ashmead) (Hymenoptera: Braconidae) em pomar diversificado em Conceição do Almeida, BA. Neotrop. Entomol. 2005, 34, 799-805.

132. Alvarenga, C.D.; Brito, E.S.; Lopes, E.N.; Silva, M.A.; Alves, D.A.; Matrangolo, C.A.R.; Zucchi, R.A. Introdução e recuperação do parasitóide exótico Diachasmimorpha longicaudata (Ashmead) (Hymenoptera: Braconidae) em pomares comerciais de goiaba no norte de Minas Gerais. Neotrop. Entomol. 2005, 34, 133136.

133. Blanco-Metzler, H.; Morena-Montoya, R. Biological Control in Costa Rica. In Biological Control in Latin America and the Caribbean: Its Rich History and Bright Future; van Lenteren, J.C., Bueno, V.H.P., Luna, M.G., Colmenarez, Y.C., Eds.; CAB International: Wallingford, UK, 2019; pp.162-175.

134. Rivera García, S. Estudio de la mosca del meditarráneo Ceratitis capitata en El Salvador. CENTA Div. Invest. Agrop. 1977, 17, 1-9.

135. Vayssières, J.-F.; Cayol, J.P.; Caplong, P.; Séguret, J.; Midgarden, D.; Van Sauers-Muller, A.; Zucchi, R.; Uramoto, K.; Malavasi, A. Diversity of fruit fly (Diptera: Tephritidae) species in French Guiana: Their main host plants and associated parasitoids during the period 1994-2003 and prospects for management. Fruits 2013, 68, 219-243.

136. López-Martínez, V. First record of Diachasmimorpha longicaudata (Ashmead) 1905 (Hymenoptera: Braconidae) parasitizing the papaya fruit fly Toxotrypana curvicauda Gerstaecker 1860. Entomotropica 2005, 20, 121-123.

137. Montoya, P.; Liedo, P.; Benrey, B.; Cancino, J.; Barrera, J.F.; Sivinski, J.; Aluja, M. Biological control of Anastrepha spp. (Diptera: Tephritidae) in mango orchards through augmentative releases of Diachasmimorpha longicaudata (Ashmead) (Hymenoptera: Braconidae). Biol. Control 2000, 18, 216-224. 
138. Montoya, P.; Cancino, J.; Zenil, M.; Gómez, E.; Villaseñor, A. Parasitoid releases in the control of Ceratitis capitata (Diptera: Tephritidae) outbreaks in coffee growing zones of Chiapas, Mexico. Vedalia 2005, 12, 8589.

139. Vaughan, M.A. International biocontrol cooperation within Latin America. In Opportunities for Implementation of Biocontrol in Latin America, Proccedings of the IOBC Workshop, Buenos Aires; Coulson, J.R., Zapater, M.C., Eds.; SRNT, IOBC: Buenos Aires, Argentina, 1992; pp. 7-38.

140. Salmerón Delgado, A.L. Hymenoptera parasitoides asociados a Anastrepha spp (Diptera: Tephritidae) en frutales de San Francisco Libre y El Jicaral, Nicaragua. Master's Thesis, Universidad Nacional Agraria, Facultad de Agronomia, Managua, Nicaragua, 2016; 48p.

141. Beingolea, O.D.G. El control biológico en el Perú: Avances, perspectivas, aplicación y limitaciones. In El Control Biológico en América Latina; Zapater, M.C., Ed.; SRNT, IOBC: Buenos Aires, Argentina, 1996; pp. 7191.

142. Mujica, M.; Whu, N. Biological Control in Perú. In Biological Control in Latin America and the Caribbean: Its Rich History and Bright Future; van Lenteren, J.C.; Bueno, V.H.P.; Luna, M.G.; Colmenarez, Y.C.; Eds.; CAB International: Wallingford, UK, 2019; pp. 369-389.

143. Hentze, F.; Mata, R.; Urbina, N.A Central American Program for fruit fly control. In Fruit Flies: Biology and Management; Aluja, M., Liedo, P., Eds.; Springer: New York, NY, USA, 1993; pp. 449-454.

144. Sivinski, J.M.; Calkins, C.O.; Baranowski, R.; Harris, D.; Brambila, J.; Diaz, J.; Burns, R.E.; Holler, T.; Dodson, G. Suppression of a caribbean fruit fly (Anastrepha suspensa (Loew)) (Diptera: Tephritidae) population through augmentative releases of the parasitoid Diachasmimorpha longicaudata (Ashmead) (Hymenoptera: Braconidae). Biol. Control 1996, 16, 177-185.

145. Albornoz Medina, P.; van Nieuwenhove, G.; Bezdjian, L.P.; Schliserman, P.; Fidelis-Marinho, C.; Ovruski, S.M. Mortality by parasitization in the association between Diachasmimorpha tryoni (Hymenoptera: Braconidae) and Ceratitis capitata (Diptera: Tephritidae) under field-cage conditions. Nat. Sci. 2014, 6, 12671274.

146. Sivinski, J.; Piñeero, J.; Aluja, M. The distributions of parasitoids (Hymenoptera) of Anastrepha fruit flies (Diptera: Tephritidae) along an altitudinal gradient in Veracruz, Mexico. Biol. Control 2000, 18, $258-269$.

147. Cancino, J.L.; López, E.; Aguilar, C.E. Liberaciones inundativas de parasitoides como método alternativo de control de Ceratitis capitata en fincas cafetaleras en el Soconusco, Chiapas, México. In Actas de la Primera Conferencia Internacional sobre Café Orgánico en México; Universidad Autónoma de Chapingo: Texcoco, Mexico, 1995; pp. 51-53.

148. Jimenez-Jimenez, E. Fruit flies and their natural enemies. Fitófilo 1956, 16, 4-11.

149. Wong, T.T.Y.; Ramadan, M.M.; McInnis, D.O.; Mochizuki, N.; Nishimoto, J.J.; Herr, J.C. Augmentative releases of Diachasmimorpha tryoni (Hymenoptera: Braconidae) to suppress a Mediterranean fruit fly (Diptera: Tephritidae) population in Kula, Maui, Hawaii. Biol. Control 1991, 1, 2-7.

150. Bokonon-Ganta, A.H.; McQuate, G.T.; Messing, R.H. Natural establishment of a parasitoid complex on Bactrocera latifrons (Diptera: Tephritidae) in Hawaii. Biol. Control 2007, 42, 365-373.

151. Paranhos, B.J.; Nava, D.E.; Malavasi, A. Biological control of fruit flies in Brazil. Pesq. Agropec. 2019, 54, e26037.

152. Zenil, M.; Liedo, P.; Williams, T.; Valle, J.; Cancino, J.; Montoya, P. Reproductive biology of Fopius arisanus (Hymenoptera: Braconidae) on Ceratitis capitata and Anastrepha spp. (Diptera: Tephritidae). Biol. Control 2004, 29, 169-178.

153. Montoya, P.; Suarez, A.; Lopez, F.; Cancino, J. Fopius arisanus oviposition in four Anastrepha fruit fly species of economic importance in Mexico. BioControl 2009, 54, 437-444.

154. Purcell, M.F. Contribution of biological control to integrated pest management of tephritid fruit flies in the tropic and subtropics. Integr. Pest Manag. Rev. 1998, 3, 63-83.

155. Harris, E.J.; Bautista, R.C.; Vargas, R.I.; Jang, E.B.; Eitam, A.; Leblanc, L. Suppression of melon fly (Diptera: Tephritidae) populations with releases of Fopius arisanus and Psyttalia fletcheri (Hymenoptera: Braconidae) in North Shore Oahu, HI, USA. BioControl 2010, 55, 593-599.

156. López, M.; Sivinski, J.; Rendon, P.; Holler, T.; Bloem, K.; Copeland, R.; Trostle, M.; Aluja, Colonization of Fopius ceratitivorus, a newly discovered african egg-pupal parasitoid (Hymenoptera: Braconidae) of Ceratitis capitata (Diptera: Tephritidae). Fla. Entomol. 2003, 86, 53-60. 
157. Bokonon-Ganta, A.H.; Ramadan, M.M.; Messing, R.H. Insectary production and synopsis of Fopius caudatus (Hymenoptera: Braconidae), parasitoid of tephritid fruit flies indigenous to Africa. J. Asia Pac. Entomol. 2019, 22, 359-371.

158. Bokonon-Ganta, A.H.; Ramadan, M.M.; Messing, R.H. Reproductive biology of Fopius ceratitivorus (Hymenoptera: Braconidae), an egg-larval parasitoid of the Mediterranean fruit fly, Ceratitis capitata (Diptera: Tephritidae). Biol. Control 2007, 41, 361-367.

159. Baranowski, R.; Glenn, H.; Sivinski, J. Biological control of the caribbean fruit fly (Diptera: Tephritidae). Fla. Entomol. 1993, 76, 245-251.

160. Clarke, A.R. Biology and Management of Bactrocera and Related Fruit Flies; CSIRO Publishing: Victoria, Australia, 2019; 272p.

161. Clausen, C.P. Biological control of fruit flies. J. Econ. Entomol. 1956, 49, 176-178.

162. Wharton, R.A.; Gilstrap, F.E. Key to and status of Opiinae braconid (Hymenoptera: Braconidae) parasitoid used in biological control of Ceratitis and Dacus s.l. (Diptera: Tephritidae). Ann. Entomol. Soc. Am. 1983, 76, 721-741.

163. Hernandez, J.R. Biological control in Costa Rica. In El Control Biológico en América Latina; Zapter, M.C., Ed.; SRNT, IOBC: Buenos Aires, Argentina, 1996; pp. 35-40.

164. Wharton, R.A. Classical biological control of fruit infesting Tephritidae. In World Crop Pests. Fruit Flies: Their Biology, Natural Enemies, and Control; Robinson, A., Harper, G., Eds.; Elsevier: Amsterdam, The Netherlands, 1989; pp. 303-313.

165. Sime, K.R.; Daane, K.M.; Messing, R.H.; Johnson, M.W. Comparison of two laboratory cultures of Psyttalia concolor (Hymenoptera: Braconidae), as a parasitoid of the olive fruit fly. Biol. Control 2006, 39, 248-255.

166. Yokoyama, V.Y.; Wang, X.G.; Aldana, A.; Cáceres, C.E., Rendón, P.A., Johnson, M.W.; Daane, K.M. Performance of Psyttalia humilis (Hymenoptera: Braconidae) reared from irradiated host on olive fruit fly (Diptera: Tephritidae) in California. Environ. Entomol. 2012, 41, 497-507.

167. Daane, K.M.; Wang, X.G.; Nieto, D.J.; Pickett, C.H.; Hoelmer, K.A.; Blanchet, A.; Johnson, M.W. Classic biological control of olive fruit fly in California, USA: Release and recovery of introduced parasitoids. BioControl 2015, 60, 317-330.

168. Daane, K.M.; Sime, K.R.; Wang, X.G.; Nadel, H.; Johnson, M.W.; Walton, V.M. Psyttalia lounsburyi (Hymenoptera: Braconidae), potential biological control agent for the olive fruit fly in California. Biol. Control 2008, 44, 78-89.

169. Sime, K.R.; Daane, K.M.; Kirk, A.A.; Andrews, J.W.; Johnson, M.W.; Messing, R.H. Psyttalia ponerophaga (Hymenoptera: Braconidae) as a potential biological control agent of olive fruit fly Bactrocera oleae (Diptera: Tephritidae) in California. Bull. Entomol. Res. 2007, 97, 233-242.

170. Daane, K.M.; Johnson, M.W.; Pickett, C.H.; Sime, K.R.; Wang, X.G.; Nadel, H.; Andrews, J.W.; Hoelmer, K.A. Biological controls investigated to aid management of the olive fruit fly in California. Calif. Agric. 2011, $65,21-28$.

171. Bascope, J.B. Resumen histórico del control biológico de Bolivia. In Control Biológico en el Cono Sur; Belarmino, L.C., Carneiro, R.M.D.G., Puignau, J.P., Eds.; IICA-PROCISUR/EMBRAPA-CPACT: Gramado, Brasil, 1994; pp. 141-148.

172. Wang, X.G., Messing, R.H. Two different life-history strategies determine the competitive outcome between Dirhinus giffardii (Chalcididae) and Pachycrepoideus vindemmiae (Pteromalidae), ectoparasitoids of cyclorrhaphous Diptera. Bull. Entomol. Res. 2004, 94, 473-480.

173. Jimenez-Jimenez, E. El Syntomosphyrum indicum silv., un enemigo natural de las moscas de la fruta. Fitofilo 1958, 21, 25-30.

174. Jimenez-Jimenez, E. The Enemigo Natural Complex for the control of insects that constitute plagues in the Mexican Republic. Rev. Chapingo 1959, 12, 191-208.

175. Fernandes, E.C.; Macêdo Souza, M.; Alves Souza, M.I.; Nunes Felipe, A.G.; Araujo, E.L. Rearing technique and demographic parameters of Tetrastichus giffardianus Silvestri (Hymenoptera: Eulophidae). Semin-Cienc. Agrar. Londrina 2019, 40, 2201-2208.

176. Jimenez-Jimenez, E. Avances y resultados del control biológico en México. Fitófilo 1963, 38, $34-37$.

177. Salles, L.A.B. Parasitismo de Anastrepha fraterculus (Wied.) (Diptera: Tephritidae) por Hymenoptera, na região de Pelotas, RS. Pesqui. Agropecu. Bras. 1996, 31, 769-774.

178. Nishida, T. Natural enemies of the melon fly, Dacus curcurbitae Coq. in Hawaii. Ann. Entomol. Soc. Am. 1955, $48,171-178$. 
179. Clausen, C.P.; Clancy, D.W.; Chock, Q.C. Biological Control of the Oriental Fruit Fly (Dacus Dorsalis Hendel) and other Fruit Flies in Hawaii; Agricultural Research Service; US Dept. of Agriculture: Washington, DC, USA, 1965; 108p.

180. Allen, W.R.; Hagley, E.A.C. Epigeal arthropods as predators of mature larvae and pupae of the apple maggot (Diptera: Tephritidae). Environ. Entomol. 1990, 19, 309-312.

181. Monteith, L.G. Status of predators of the adult of apple maggot, Rhagolethis pomonella (Diptera: Tephritidae) in Ontario. Can. Ent. 1972, 104, 257-262.

182. Garcia, F.R.M. Zoologia Agrícola: Manejo Ecológico de Pragas, 4th ed.; Editora Rígel: Porto Alegre, Brazil, 2014; p. 256.

183. Gobbi, P.C.; Nunes, A.M.; Zefa, E.; Garcia, F.R.M. Influence of wing bands and behavior of Anastrepha fraterculus (Diptera: Tephritidae) by the presence of Megafreya sutrix (Araneae: Salticidae). An. Acad. Bras. Ciênc. 2020, 92, e20181306.

184. Rao, D.; Díaz-Fleischer, F. Characterisation of predator-directed displays in tephritid flies. Ethology 2012, 118, 1165-1172.

185. Rao, D.; Aguilar-Argüello, S.; Montoya, P.; Díaz-Fleischer, F. The effect of irradiation and mass rearing on the anti-predator behaviour of the Mexican fruit fly, Anastrepha ludens (Diptera: Tephritidae). Bull. Entomol. Res. 2014, 104, 176-181.

186. Ramírez, L.A. Macrocheles sp. (Acari: Macrochelidae): Nuevo ácaro predador de Anastrepha suspensa (Diptera: Tephritidae) en Cuba. Rev. Prot. Veg. 1987, 2, 37-41.

187. Santos, M.D.; Hanel, A.; Mastrangelo, T.; Lopes, L.A.; Silva, E.S.; Morales, G.J. Predação e oviposição de Macrocheles roquensis (Acari: Macrochelidae) alimentado com Ceratitis capitata (Diptera, Tephritidae). In Proceedings of the 15th Simpósio de Controle Biológico, Ribeirão Preto, SP, Brazil, 4- June 2017; pp. 434225.

188. Monteith, L.G. Crickets as predators of the apple maggot, Rhagoletis pomonella (Diptera: Tephritidae). Can. Ent. 1971, 103, 52-58.

189. Hennessey, M.K. Predation on Caribbean Fruit Fly Prepupae and Pupae in Guava and Carambola Grove; USDA, ARS, Tektran: Miami, FL, USA, 1998.

190. Renkema, J.M.; Manning, P.; Cutler, G.C. Predation of lowbush blueberry insect pests by ground beetles (Coleoptera: Carabidae) in the laboratory. J. Pest Sci. 2013, 86, 525-532.

191. Renkema, J.M.; Lynch, D.H.M.; Cutler, G.C.; MacKenzie, K.; Walde, S.J. Predation by Pterostichus melanarius (Illiger) (Coleoptera: Carabidae) on immature Rhagoletis mendax Curran (Diptera: Tephritidae) in semi-field and field conditions. Biol. Control 2012, 60, 46-53.

192. Silva, A.; Gonçalves, C.R.; Galvão, D.M.; Gonçalves, A.J.L.; Gomes, J.; Silva, M.N.; Simoni, L.M. Quarto Catálogo dos Insetos que Vivem nas Plantas do Brasil: Seus Parasitos e Predadores; Ministério da Agricultura: Rio de Janeiro, Brazil, 1968; 622p.

193. Aluja, M. Bionomics and management of Anastrepha. Ann. Rev. Entomol. 1994, 39, 155-173.

194. Thomas, D.B. Predation on the Soil Inhabiting Stages of the Mexican Fruit Fly. Southwest. Entomol. 1995, 20, 61-71.

195. Eskafi, F.M.; Kolbe, M.M. Predation on larval and pupal Ceratitis capitata (Diptera: Tephritidae) by the ant Solenopsis geminate (Hymenoptera: Formicidae) and others predators in Guatemala. Environ. Entomol. 1990, 19, 148-153.

196. Dor, A.; Valle-Mora, J.; Rodríguez-Rodriguez, S.E.; Liedo, P. Predation of Anastrepha ludens (Diptera: Tephritidae) by Norops serranoi (Reptilia: Polychrotidae): Functional response and evasion ability. Environ. Entomol. 2014, 43, 706-715.

197. Thomas, D.B. Survivorship of the Pupal Stages of the Mexican Fruit Fly Anastrepha ludens (Loew) (Diptera: Tephritidae) in an Agricultural and a Nonagricultural Situation. J. Entomol. Sci. 1993, 28, 350-362.

198. Radeghieri, P. Cameraria ohridella (Lepidoptera Gracillariidae) predation by Crematogaster scutellaris (Hymenoptera Formicidae) in Northern Italy (Preliminary note). Bull. Insectol. 2004, 57, 63-64.

199. Vargas, R.I.; Long, J.; Miller, N.W.; Delate, K.; Jackson, C.G.; Uchida, G.K.; Bautista, R.C.; Harris, E.J. Releases of Psyttalia fletcheri (Hymenoptera: Braconidae) and sterile flies to suppress melon fly (Diptera: Tephritidae) in Hawaii. J. Econ. Entomol. 2004, 97, 1531-1539.

200. Vargas, R.I.; Stark, J.D.; Uchida, G.; Purcell, M. Opiine parasitoids (Hymenoptera, Braconidae) of Oriental fruit fly (Diptera, Tephritidae) on Kauai Island, Hawaii Islandwide, relative abundance and parasitism rates in wild and orchard guava habitats. Environ. Entomol. 1993, 22, 246-253. 
201. Wang, X.G.; Johnson, M.W.; Daane, K.M., Nadel, H. High summer temperatures affect survival and reproduction of olive fruit fly (Diptera: Tephritidae). Environ. Entomol. 2009, 38, 1496-1504.

202. Yokoyama, V.Y.; Rendón, P.A.; Sivinski, J. Psyttalia cf. concolor (Hymenoptera: Braconidae) for biological control of olive fruit fly (Diptera: Tephritidae) in California. Environ. Entomol. 2008, 37, 764-773.

203. Sime, K.R.; Daane, K.M.; Wang, X.G.; Johnson, M.W.; Messing, R.H. Evaluation of Fopius arisanus as a biological control agent for the olive fruit fly in California. Agri. For. Entomol. 2008, 10, 423-431.

204. Yokoyama, V.Y.; Rendón, P.A.; Wang, X.G.; Opp, S.B.; Johnson, M.W.; Daane, K.M. Response of Psyttalia humilis (Hymenoptera: Braconidae) to olive fruit fly (Diptera: Tephritidae) and conditions in California olive orchards. Environ. Entomol. 2011, 40, 315-323.

205. Daane, K.M.; Wang, C.P.; Pickett, A.; Blanchet, D.; Nieto, K.A.; Hoelmer, M.C.; Bon, M.V.; Smith, L. Biological Control of Olive Fruit Fly in - Release, Establishment and Impact of Psyttalia lounsburyi and Psyttalia humilis. In Proceedings of the 5th International Symposium on Biological Control of Arthropods, Langkawi, Malaysia, 11-15 September 2017; Mason, P.G., Gillespie, D.R., Vincent, C., Eds.; CAB International: Wallingford, UK, 2017; pp. 156-158.

206. Chardonnet, F.; Blanchet, A.; Hurtrel, B.; Marini, F.; Smith, L. Mass-rearing optimization of the parasitoid Psyttalia lounsburyi for biological control of the olive fruit fly. J. Appl. Entomol. 2019, 143, 277-288.

207. Vargas, R.I.; Peck, S.L.; McQuate, G.T.; Jackson, C.G.; Stark, J.D.; Armstrong, J.W. Potential for areawide integrated management of Mediterranean fruit fly (Diptera: Tephritidae) with a Braconid parasitoid and a novel bait spray. J. Econ. Entomol. 2001, 94, 817-825.

208. Wong, T.T.Y.; Ramadan, M.M. Mass rearing of larval parasitoid (Hymenoptera: Braconidae; Opiinae) of tephritid flies (Diptera: Tephritidae) in Hawaii. In Advances in Insect Rearing for Research and Pest Management; Anderson, T.E., Leppla, N., Eds.; Westview Press Inc.: Boulder, CO, USA, 1992; pp. 405-426.

209. Messing, R.H.; Klungness, L.M.; Purcell, M.F.; Wong, T.T. Quality control parameters of reared Opiinae parasitoids used in augmentative biological control of tephritid fruit flies in Hawaii. Biol. Control 1993, 3, 140-147.

210. Bautista, R.C.; Mochizuki, N.; Spencer, J.P.; Harris, E.J.; Ichimura, D.M. Mass-rearing of the tephritid fruit fly parasitoid Fopius arisanus (Hymenoptera: Braconidae). Biol. Control 1999, 15, 137-144.

211. Messing, R.H. Status and needs of biological control research for tephritid flies. In Economic Fruit Flies: A World Assessment of Their Biology and Management; McPheron, B.A., Steck, G., Eds.; St. Lucie Press: Delray Beach, FL, USA, 1995; pp. 365-367.

212. Wong, T.T.Y.; Ramadan, M.M.; Herr, J.; McInnis, D.O. Suppression of a Mediterranean fruit fly (Diptera: Tephritidae) population with concurrent parasitoid and sterile fly release in Kula, Maui, Hawaii. J. Econ. Entomol. 1992, 85, 1671-168.

213. Kroder, S.; Messing, R.H. A new parasitoid from Kenya, Fopius ceratitivorus, complements the extant parasitoid guild attacking Mediterranean fruit fly in Hawaii. Biol. Control 2010, 53, 223-229.

214. Wharton, R.A.; Trostle, M.K.; Messing, R.H.; Copeland, R.S.; Kimani-Njogu, S.W.; Lux, S.; Overholt, W.A.; Mohamed, S.; Sivinski, J. Parasitoids of medfly, Ceratitis capitata, and related tephritids in Kenyan coffee: A predominantly koinobiont assemblage. Bull. Entomol. Res. 2000, 90, 517-526.

215. Messing, R.H.; Wright, M.G. Biological control of invasive species: Solution or pollution? Front. Ecol. Environ. 2006, 4, 132-140.

216. Wang, X.G.; Bokonon-Ganta, A.H.; Ramadan, M.M.; Messing, R.H. Egg-larval opiine parasitoids (Hym., Braconidae) of tephritid fruit fly pests do not attack the flower head-feeder Trupanea dubautiae (Dipt., Tephritidae). J. Appl. Entomol. 2004, 128, 716-722.

217. Bokonon-Ganta, A.H.; Ramadan, M.M.; Wang, X.G.; Messing, R.H. Biological performance and potential of Fopius ceratitivorus (Hymenoptera: Braconidae), an egg-larval parasitoid of tephritid fruit flies newly imported to Hawaii. Biol. Control 2005, 33, 238-247.

218. Cancino, J.; Ruiz, L.; López, E.; Aguilar, E.; Gálvez, C.; Montoya, P.; Liedo, P. Suppression of Ceratitis capitata (Wied.) (Diptera: Tephritidae) populations in coffee in the Mexico-Guatemala border region through the augmentative releases of Diachasmimorpha longicaudata (Ashmead) (Hymenoptera: Braconidae). Biocontrol Sci. Technol. 2019, 29, 822-826.

219. Cancino, J.; Ruíz, L.; Viscarret, M.; Sivinski, J.; Hendrichs, J. Application of nuclear techniques to improve the mass production and management of fruit fly parasitoids. Insects 2012, 3, 1105-1125.

220. Wharton, R.A. Changes in nomenclature and classification of some Opiine Braconidae (Hymenoptera). Proc. Entomol. Soc. Wash. 1987, 89, 61-73. 
221. Cancino, J.; Ruíz, L.; López, P.; Sivinski, J. The suitability of Anastrepha spp. and Ceratitis capitata larvae as hosts of Diachasmimorpha longicaudata and Diachasmimorpha tryoni: Effects of host age and radiation dose and implications for quality control in mass rearing. Biocontrol Sci. Technol. 2009, 19, 81-94.

222. Montoya, P.; Cancino, J.; Ruiz, L. Packing of fruit fly parasitoids for augmentative releases. Insects 2012, 3, 889-899.

223. Enkerlin, D.; Cancino, J.; Guillén, J.; Martínez, L. Evaluaciones del efecto de las liberaciones de parasitoides sobre poblaciones de moscas de la fruta del género Anastrepha (Diptera: Tephritidae) en Mazapa de Madero, Chiapas. In Proceedings of the XIII Reunión Nacional de Control Biológico, AMBC, Mexico D.F., México, 12-17 March 1990, Unpublished.

224. Montoya, P.; Pérez-Lachaud, G.; Liedo, P. Superparasitism in the fruit fly parasitoid Diachasmimorpha longicaudata (Hymenoptera: Braconidae) and the Implications for mass rearing and augmentative release. Insects 2012, 3, 900-911.

225. Cancino, J.; López-Arriaga, F.; Montoya, P. Packaging conditions for the field release of the fruit fly parasitoid Diachasmimorpha longicaudata (Hymenoptera: Braconidae). Austral Entomol. 2017, 56, 261-267.

226. Aluja, M.; Rull, J. Managing pestiferous Fruit Flies (Diptera: Tephritidae) through environmental manipulation. In Biorational Tree Fruit Pest Management; Aluja, M., Leskey, T., Vincent, C., Eds.; CAB International: Wallingford, UK, 2009; pp. 171-213.

227. Cancino, J.; Liedo, P.; Ruiz, L.; López, G.; Montoya, P.; Barrera, J.F.; Sivinski, J.; Aluja, M. Discrimination by Coptera haywardi (Hymenoptera: Diapriidae) of hosts previously attacked by conspecifics or by the larval parasitoid Diachasmimorpha longicaudata (Hymenoptera: Braconidae). Biocontrol Sci. Technol. 2012, 22, 899914.

228. Miranda, M.; Sivinski, J.; Rull, J.; Cicero, L.; Aluja, M. Niche breadth and interspecific competition between Doryctobracon crawfordi and Diachasmimorpha longicaudata (Hymenoptera: Braconidae), native and introduced parasitoids of Anastrepha spp. fruit flies (Diptera: Tephritidae). Biol. Control 2015, 82, 86-95.

229. Cancino, J.; Ruíz, L.; López, P.; Sivinski, J.; Gálvez, F.O.; Aluja, M. Rearing of five hymenopterous larvalprepupal (Braconidae, Figitidae) and three pupal (Diapriidae, Chalcidoidea, Eurytomidae) native parasitoids of the genus Anastrepha (Diptera: Tephritidae) on irradiated A. ludens larvae and pupae. Biocontrol Sci. Technol. 2009, 19, 193-209.

230. Cancino, J.; Pérez, B.; Johnson, A.C.; Reynolds, O.L. Parasitoids are choosy: Increase in the capacity to discriminate parasitised tephritid pupae by Coptera haywardi. BioControl 2019, 64, 357-366.

231. Cancino, J., López-Arriaga, F.; Gálvez, C.; Verdugo, E.; Solis, E.; Moreno, F.; Quintero-Fong, L.; OrozcoDavila, D. Mechanical sorting of young Anastrepha ludens Tapachula-7 pupae, a host for Coptera haywardi, negatively affects sterile male quality. BioControl 2019, 64, 563-572.

232. Burns, R.E.; Diaz, J.D.; Holler, T.C. Inundative release of the parasitoid Diachasmimorpha longicaudata for the control of the caribbean fruit fly, Anastrepha suspensa. In Fruit Fly Pest: A World Assessment of Their Biology and Management; McPheron, B.A., Steck, G.J., Eds.; St. Lucie Press: DelRay Beach, FL, USA, 1996; pp. 377381.

233. Paranhos, B.J.; Sivinski, J.; Stuhl, C.; Holler, T.; Aluja, M. Intrinsic competition and competitor-free-space influence the coexistence of parasitoids Hymenoptera: Braconidae: Opiinae of Neotropical tephritidae (Diptera). Environ. Entomol. 2013, 42, 717-723.

234. Groth, M.Z.; Loeck, A.E.; Nörnberg, S.D.; Bernardi, D.; Nava, D.E. Biology of Fopius arisanus (Hymenoptera: Braconidae) in two species of fruit flies. J. Insect Sci. 2016, 16, 96.

235. Gonçalves, R.S.; Nava, D.E.; Pereira, H.C.; Lisbôa, H.; Grützmacher, A.D.; Valgas, R.A. Biology and fertility life table of Aganaspis pelleranoi (Hymenoptera: Figitidae) in larvae of Anastrepha fraterculus and Ceratitis capitata (Diptera: Tephritidae). An. Entomol. Soc. Am. 2013, 106, 791-798.

236. Gonçalves, R.S.; Andreazza, F.; Lisbôa, H.; Grützmacher, A.D.; Valgas, R.A.; Manica-Berto, R.; Nava, D.E. Basis for the development of a rearing technique of Aganaspis pelleranoi (Hymenoptera: Figitidae) in Anastrepha fraterculus (Tephritidae: Diptera). J. Econ. Entomol. 2016, 109, 1094-1101.

237. Poncio, S.; Nunes, A.M.; Gonçalves, R.S.; Lisboa, H.; Manica-Berto, R.; Garcia, M.S.; Nava, D.E. Biology of Doryctobracon brasiliensis at different temperatures: Development of life table and determining thermal requirements. J. Appl. Entomol. 2016, 140, 775-785.

238. Poncio, S.; Nunes, A.M.; Gonçalves, R.S.; Lisboa, H.; Manica-Berto, R.; Garcia, M.S.; Nava, D.E. Strategies for establishing a rearing technique for the fruit fly parasitoid: Doryctobracon brasiliensis (Hymenoptera: Braconidae). J. Econ. Entomol. 2018, 111, 1087-1095. 
239. van Nieuwenhove, G.A.; Bezdjian, L.P.; Schliserman, P.; Aluja, M.; Ovruski, S.M. Combined effect of larval and pupal parasitoid use for Anastrepha fraterculus (Diptera: Tephritidae) control. Biol. Control 2016, 95, 94102.

240. Núñez-Campero, S.R.; Aluja, M.; Rull, J.; Ovruski, S.M. Comparative demography of three neotropical larval-prepupal parasitoid species associated with Anastrepha fraterculus (Diptera: Tephritidae). Biol. Control 2014, 69, 8-17.

241. Buonocore Biancheri, M.J.; Suárez, L.; Bezdjian, L.P.; van Nieuwenhove, G.A.; Rull, J.; Ovruski, S.M. Response of two parasitoid species (Hymenoptera: Braconidae, Figitidae) to tephritid host and host food substrate cues. J. Appl. Entomol. 2019, 143, 344-356.

242. Boller, E.F.; Prokopy, R.J. Bionomics and management of Rhagoletis. Annu. Rev. Entomol. 1976, 21, $223-246$.

243. Aliniazee, M.T.; Croft, B.A. Biological control in deciduous fruit crops. In Handbook of Biological Control: Principles and Applications; Bellows, T.S.; Fisher, T.W. Eds.; Academic Press: San Diego, NY, USA, 1999; pp. 743-759.

244. Rosenheim, J.A.; Kaya, H.K.; Ehler, L.E.; Marois, J.J.; Jaffee, B.A. Intraguild predation among biological control agents: Theory and evidence. Biol. Control 1995, 5, 303-335.

(C) 2020 by the authors. Licensee MDPI, Basel, Switzerland. This article is an open access article distributed under the terms and conditions of the Creative Commons Attribution (CC BY) license (http://creativecommons.org/licenses/by/4.0/). 